\title{
A biosensor for the direct visualization of auxin
}

https://doi.org/10.1038/s41586-021-03425-2

Received: 2 October 2018

Accepted: 5 March 2021

Published online: 7 April 2021

\section{Open access}

Check for updates

\section{Ole Herud-Sikimić, ${ }^{1,5}$, Andre C. Stiel ${ }^{1,2,5}$, Martina Kolb ${ }^{1,5}$, Sooruban Shanmugaratnam, ${ }^{1,3}$ Kenneth W. Berendzen ${ }^{4}$, Christian Feldhaus ${ }^{1}$, Birte Höcker ${ }^{1,3 凶}$ \& Gerd Jürgens ${ }^{1 凶}$}

One of the most important regulatory small molecules in plants is indole-3-acetic acid, also known as auxin. Its dynamic redistribution has an essential role in almost every aspect of plant life, ranging from cell shape and division to organogenesis and responses to light and gravity ${ }^{1,2}$. So far, it has not been possible to directly determine the spatial and temporal distribution of auxin at a cellular resolution. Instead it is inferred from the visualization of irreversible processes that involve the endogenous auxin-response machinery ${ }^{3-7}$; however, such a system cannot detect transient changes. Here we report a genetically encoded biosensor for the quantitative in vivo visualization of auxin distribution. The sensor is based on the Escherichia coli tryptophan repressor ${ }^{8}$, the binding pocket of which is engineered to be specific to auxin. Coupling of the auxin-binding moiety with selected fluorescent proteins enables the use of a fluorescence resonance energy transfer signal as a readout. Unlike previous systems, this sensor enables direct monitoring of the rapid uptake and clearance of auxin by individual cells and within cell compartments in planta. By responding to the graded spatial distribution along the root axis and its perturbation by transport inhibitors-as well as the rapid and reversible redistribution of endogenous auxin in response to changes in gravity vectors-our sensor enables real-time monitoring of auxin concentrations at a (sub)cellular resolution and their spatial and temporal changes during the lifespan of a plant.
The tryptophan-derived metabolite indole-3-acetic acid (IAA) has an important role in plants, triggering a multitude of developmental processes and responses to environmental cues ${ }^{1,2}$. Much progress has been made towards a mechanistic understanding of the nuclear events that transform auxin perception into transcriptional responses ${ }^{9-11}$. Other studies have investigated the basic machinery involved in the polar and non-vectorial release of auxin from the cell-which occurs through the action of PINFORMED efflux transporters and ABCB transporterswithin a tissue context, and have resulted in computer models of how canalized auxin flow mediates developmental or physiological processe $^{12-14}$. By contrast, owing to technical limitations (reviewed in ref. ${ }^{15}$ ), very little is known about the actual distribution of auxin in tissues at single-cell resolution. At present, plant biologists can use only proxies to visualize auxin distribution, such as the auxin-dependent expression of reporter genes (for example, using the systems DR5::GUS ${ }^{3}$; DR5::ER-GFP ${ }^{4}$ and DR5::NLS-3xGFP ${ }^{5}$. However, this indirect approach is characterized by latencies and can be affected by modulation of the auxin signalling machinery. More recently, IAA levels have been inferred from auxin-dependent degradation-and thus signal reduction-of fluorescent proteins linked to domain II of an IAA inhibitory protein (for example, DII-VENUS ${ }^{6}$ and R2D2 ${ }^{7}$ ). A limitation of these approaches is their irreversibility, which precludes the visualization of transient changes in auxin levels.
The ideal sensor for the visualization of auxin dynamics in planta should have the following features: first, physical interaction of the sensor with auxin should elicit a fluorescent signal in a reversible manner, so that changes in auxin concentration can be monitored; second, the sensitivity of the sensor should be sufficiently high to image the dynamic auxin distribution over time; third, the sensor should be targetable to different subcellular compartments-locations that are out of reach for the conventional proxies, which rely on gene expression or protein degradation; and fourth, the sensor should not contain components that are involved in plant metabolism or regulation, such that both interference with auxin responses and regulation of the sensor by the plant are avoided.

With these boundary conditions in mind, we developed a genetically encoded, fully reversible biosensor for in vivo imaging of auxin gradients with high spatial and temporal resolution, starting from the bacterial tryptophan repressor (TrpR). IAA resembles tryptophan: both contain an indole ring, the 3-position of which is connected to an amino acid moiety in TRP and a carboxyl group in IAA (Fig. 1a). The dimeric TrpR undergoes a conformational change upon binding TRP ${ }^{16,17}$, and fluorescent proteins fused to TrpR can relay this change, generating a fluorescence resonance energy transfer (FRET) signal as a convenient readout for in vivo measurements ${ }^{18}$ (Fig. 1b). Furthermore, TrpR exhibits low affinity towards IAA ${ }^{8}$. This makes TrpR an ideal starting point for

${ }^{1}$ Max Planck Institute for Developmental Biology, Tübingen, Germany. ${ }^{2}$ Institute for Biological and Medical Imaging, Helmholtz Zentrum Munich, German Research Center for Environmental Health, Munich, Germany. ${ }^{3}$ Department of Biochemistry, University of Bayreuth, Bayreuth, Germany. ${ }^{4}$ Centre for Plant Molecular Biology, University of Tübingen, Tübingen, Germany.

${ }^{5}$ These authors contributed equally: Ole Herud-Sikimić, Andre C. Stiel, Martina Kolb. ${ }^{凶}$ e-mail: birte.hoecker@uni-bayreuth.de; gerd.juergens@tuebingen.mpg.de 
a

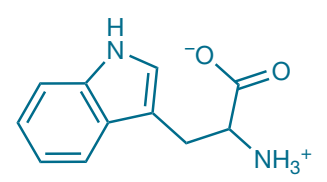

TRP

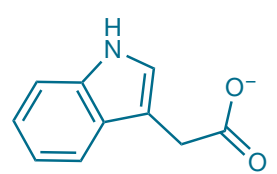

IAA
mNeonGreen
(acceptor)

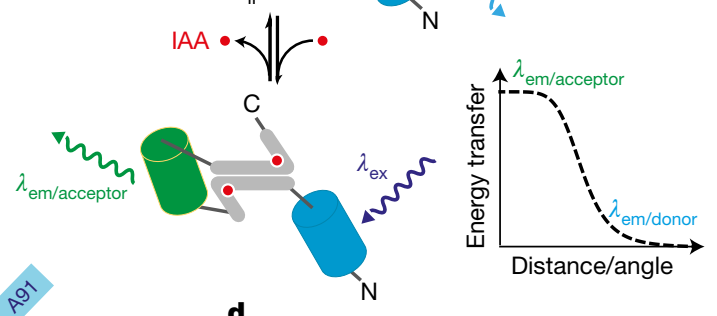

d
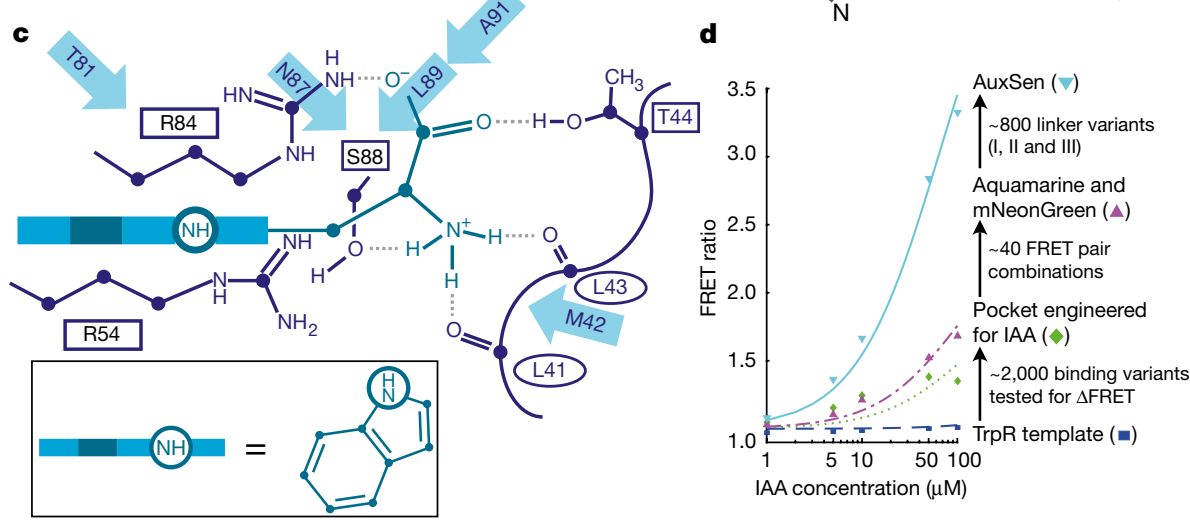

Fig. $1 \mid$ Summary of the design process. a, Chemical structures of TRP and IAA. b, Principle of the sensor design. Only in the presence of IAA (red) are the fluorophores (mNeonGreen and Aquamarine) sufficiently close and in the correct orientation for energy transfer $\left(E_{\mathrm{FRET}}\right)$. $\mathrm{N}$ and $\mathrm{C}$ represent the $\mathrm{N}$ and $\mathrm{C}$ termini of the proteins, respectively; $L$ represents the linker; and $\lambda_{\mathrm{ex}}$ and $\lambda_{\mathrm{em}}$ represent the excitation and emission wavelengths, respectively. c, Structure of the binding pocket of TrpR with ligand in side view (boxed) (modified from ref. ${ }^{8}$ ). Interactions with the side chains of R84, S88 and T44 (second TrpR chain) as well as the backbone carbonyl groups of L41 and L43 (second TrpR chain) are

developing an auxin-specific, genetically encoded FRET biosensor ${ }^{19}$. Our design efforts were aimed at improving the affinity and specificity of IAA binding, while abolishing TRP binding. We assumed a comparable binding mode for the indole ring of both TRP and IAA, and focused our design around the TrpR residues in the vicinity of the amino group of TRP (Fig.1c), aiming to improve the specificity for the carboxyl group of IAA. This selection was later expanded to include adjacent residues. Altogether, 2,000 variants were generated in successive rounds of mutagenesis and were screened for an increase in FRET signal upon the addition of IAA (Fig. 1d, Extended Data Fig. 1). Improved variants were checked for ligand specificity using a library of substances that are similar to IAA and are reportedly present in Arabidopsis (Extended Data Table 1). To confirm improvements in binding affinity, selected TrpR variants were analysed by isothermal titration calorimetry (Extended Data Table 2a). Furthermore, the structures of several variants were elucidated by X-ray crystallography, to guide mutagenesis experiments (Extended Data Table 2b, Supplementary Table 1a-h).

Our structural analysis showed that, when binding to TrpR, IAA is flipped by $180^{\circ}$ compared with TRP (Fig. 2a), with the carboxyl group of IAA facing the opening of the TrpR binding pocket (Fig. 2b). TRP is anchored by interactions with the surrounding protein residues, whereas IAA binding shows no such stabilization, which is reflected in the poor binding affinity of this interaction (Extended Data Table 2a). In engineering the auxin sensor, we identified variants that stabilize and favour this IAA-binding mode. Primarily, a serine-to-tyrosine mutation at position 88 (S88Y) was found to entirely block TRP binding owing to the bulky side chain of Y88, while simultaneously favouring IAA binding through interaction of the carboxyl group of IAA with the guanidino shown explicitly. Further residues that were mutated in this study are indicated with arrows. d, Major steps in the design of the sensor (AuxSen), and their cumulative contribution to the change in FRET ratio $(\triangle \mathrm{FRET})$ plotted against IAA concentration. Template sensor construct, TrpR-eCFP-Venus (blue squares); engineered binding pocket for IAA, TrpR(M42F/T44L/T81M/N87G/ S88Y)-eCFP-Venus (green diamonds); optimized fluorophore combination, $\operatorname{TrpR}(\mathrm{M} 42 \mathrm{~F} / \mathrm{T} 44 \mathrm{~L} / \mathrm{T} 81 \mathrm{M} / \mathrm{N} 87 \mathrm{G} / \mathrm{S} 88 \mathrm{Y})$-mNeonGreen-Aquamarine (purple triangles); AuxSen, TrpR(M42F/T44L/T81M/N87G/S88Y)-mNeonGreenAquamarine with optimised linkers I, II and III (light blue inverted triangles). group of R84 and the hydroxyl group of Y88 (Fig. 2c). The affinity for IAA was improved further by optimizing hydrophobic interactions of its indole ring with the TrpR binding pocket; to this end, the mutations T44L and T81M were incorporated in the final sensor design (Fig. 2d). During the engineering process, we also monitored the binding of indole- 3 acetonitrile (IAN) - which could potentially compete with IAA-to TrpR (Extended Data Table 2a). The binding modes of IAN and IAA are markedly similar (Extended Data Fig. 2a, b); however, mutations such as N87G exert discriminating effects through small changes in the positioning of Y88 (Extended Data Fig. 2c). Finally, we identified mutations that have no favourable effect on IAA affinity but improve the FRET readout-probably through changes in the packing, and therefore the orientation, of the attached fluorescent proteins (Extended Data Fig. 2d). We then optimized the fluorophores and the linker combinations (Extended Data Fig. 3) to yield our final sensor, which we term 'AuxSen', with the composition mNeonGreen-TrpR-Aquamarine-TrpR, in which TrpR is $\operatorname{TrpR}(\mathrm{M} 42 \mathrm{~F} / \mathrm{T} 44 \mathrm{~L} / \mathrm{T} 81 \mathrm{M} / \mathrm{N} 87 \mathrm{G} / \mathrm{S} 88 \mathrm{Y}$ ) (Figs. 1d, 2d).

In vitro, the FRET ratio of AuxSen changed by a factor of three upon treatment with $50 \mu \mathrm{M}$ IAA, which is within the range of cellular auxin concentration $\mathrm{s}^{20}$. The signal was stable at cytosolic $\mathrm{pH}$, and in the presence of reducing or oxidizing environments and all tested salt ions (Extended Data Fig. 4). The specificity of AuxSen for IAA was assessed using other indole derivatives. Among these, AuxSen showed the highest affinity for IAA; although a response was observed for other compounds, their binding affinities were reduced by around one order of magnitude (Extended Data Fig. 5). Of these indole derivatives, only IAN is present in substantial amount in plants (Extended Data Table1). However, roots show a growth response to treatment with IAN, and 

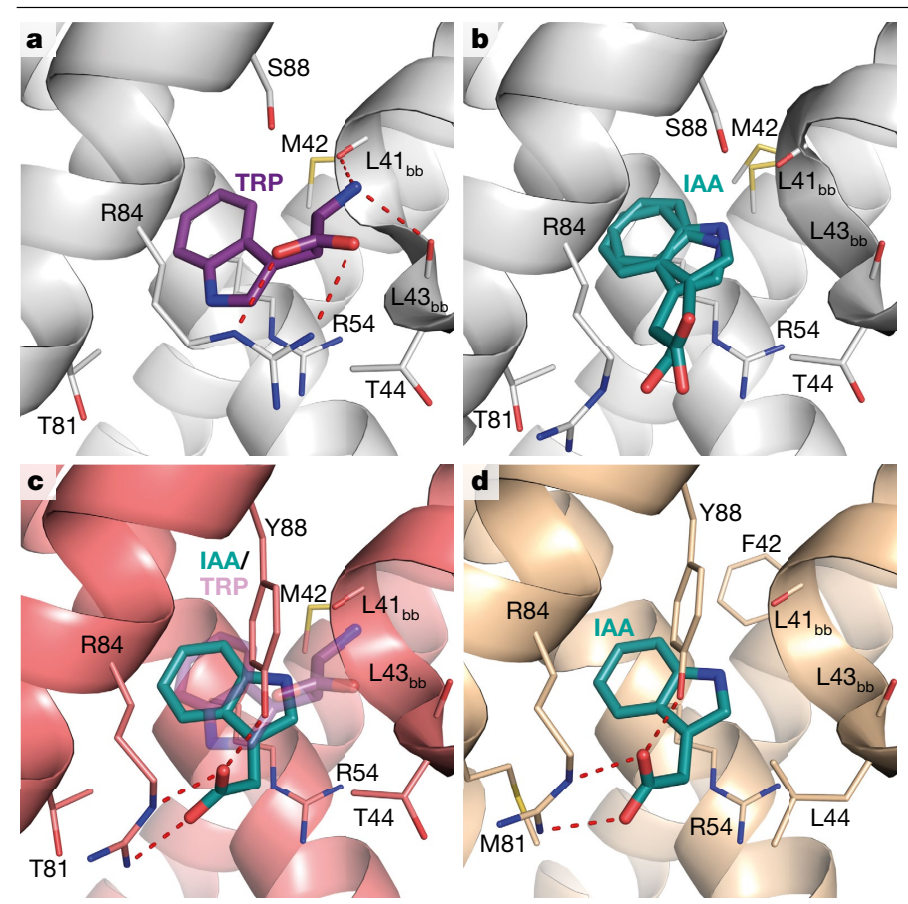

Fig. 2 Structure of AuxSen and critical steps in the engineering process. a, b, Structure of TrpR bound to the native ligand TRP (purple, Protein Data Bank (PDB) ID:1ZT9) (a) and to the design-target IAA (green) (b). IAA is rotated by $180^{\circ}$ in the binding pocket compared with TRP. Owing to a lack of stabilization when binding to $\operatorname{TrpR}$, IAA shows conformational freedom; two alternative conformations are shown. c, The mutation S88Y sterically precludes the positioning of TRP (transparent purple) in the binding site while favouring the binding of IAA.d, Structure of the final AuxSen variant (TrpR(M42F/T44L/T81M/N87G/S88Y)) bound to IAA. The ligand is firmly packed in the enhanced hydrophobic pocket of TrpR and is anchored to R84 as well as Y88, resulting in a high affinity of AuxSen for IAA. All structures are superimposed on the $C \alpha$ of residues 20-60 of both chains. Red dashed lines show polar interactions between ligand and side-chain atoms. The subscript 'bb' labels residues that have interactions of backbone atoms with the ligand.

modelling suggests that the IAA receptor $\mathrm{SCF}^{\mathrm{TIR} 1}$ could bind IAN ${ }^{21}$. Thus, IAN is probably sequestered and is therefore unlikely to interfere with auxin sensing in the plant.

As a first step to confirm the functionality of AuxSen in vivo, we expressed a nuclear-localized version of the sensor transiently from the viral 355 promoter in cell-suspension protoplast ${ }^{22}$, and quantified the FRET response by flow cytometry. The FRET ratio increased with the auxin concentration in the medium over four orders of magnitude, starting at $3 \mu \mathrm{MIAA}$ (Fig. 3a, b, Extended Data Figs. 6, 7), with the baseline FRET signal thought to reflect the endogenous level of auxin in the protoplast population. The sensitivity of the sensor could therefore be sufficient to report endogenous auxin levels.

We generated approximately 250 transgenic Arabidopsis lines bearing a dexamethasone-inducible expression system ( $p$ Bay-bar-pRPS5a-mGAL4-VP16-GR_UAS_NLS_AuxSen) integrated as a single transgene, and selected 10 lines expressing the sensor stably in the fourth generation. The strong, ubiquitously active promoter RPS5A drives expression of the yeast transcription factor Gal4p, the nuclear uptake of which is induced by dexamethasone, and the binding of Gal4p to the $U A S$ promoter results in the expression of the nuclear-localized auxin sensor. Strongly expressing lines were identified by mNeonGreen fluorescence in the root tips after dexamethasone induction overnight. To examine the response of AuxSen to auxin in planta, we treated seedlings with $10 \mu \mathrm{MIAA}$ and recorded the FRET signal over time (Fig. 3c-f). After $10 \mathrm{~min}$, the FRET signal in root nuclei had reached a maximum, and then remained constant for another $50 \mathrm{~min}$; by contrast, treatment
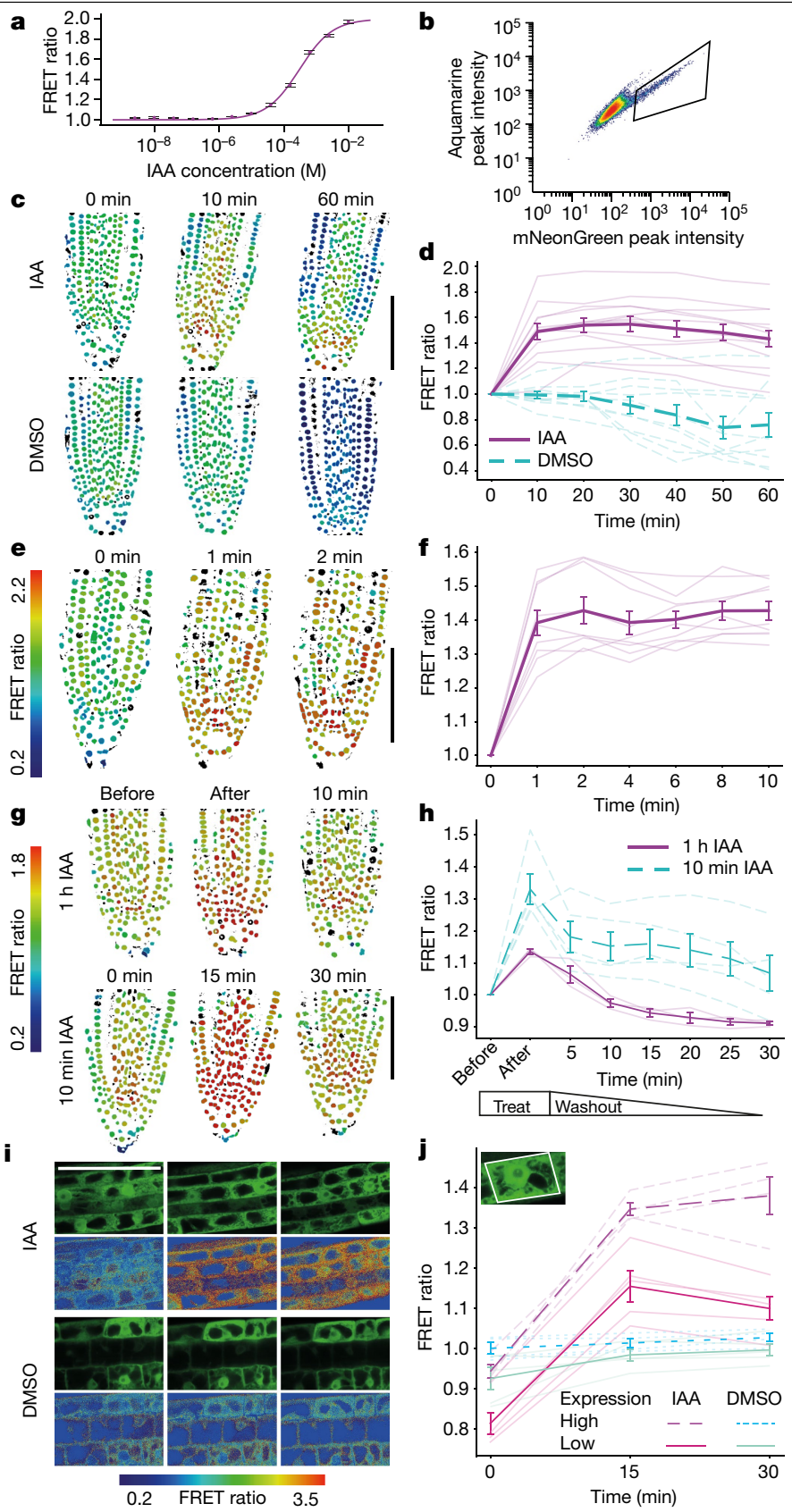

Fig. 3 | FRET ratio of AuxSen in response to auxin treatment. a, b, The FRET ratio obtained by flow cytometry in Arabidopsis protoplasts. a, Dose-response curve, normalized to the minimum FRET ratio (mean \pm s.e.m.; $n=3$ biologically independent samples). b, Baseline fluorescence intensity ('Log_Height' (a.u.)) without exogenous IAA; the relevant area is boxed (Extended Data Figs. 6, 7).c-f, Changes in the FRET ratio (colour bar) in root nuclei incubated in $10 \mu \mathrm{MIAA}$ recorded for $1 \mathrm{~h}(\mathbf{c}, \mathbf{d})$ or for $10 \min (\mathbf{e}, \mathbf{f}) . \mathbf{c}, \mathbf{e}$, Images (DMSO, control). Scale bar, $100 \mu \mathrm{m} . \mathbf{d}, \mathbf{f}$, Quantitative analyses. The thick lines represent the mean ( \pm s.e.m.), and the thin lines each represent independent single-seedling measurements ( $n=14$ (experimental), $n=10$ (control) in d, $n=9$ in f).g, h, Changes in the FRET ratio (colour bar) in root nuclei following the washout of IAA.g, Images obtained after incubation with $10 \mu$ M IAA for $1 \mathrm{~h}$ (top) or $10 \mathrm{~min}$ (bottom). Images were taken before or immediately after IAA treatment, or 10 min after the end of IAA treatment. Scale bar, $100 \mu \mathrm{m}$. h, Quantitative analysis. The thick lines represent the mean ( \pm s.e.m.), and the thin lines each represent independent single-seedling measurements $(n=5(10 \mathrm{~min}), n=3(1 \mathrm{~h})) . \mathbf{i}, \mathbf{j}$, Change in the FRET ratio of ER-localized AuxSen in response to $100 \mu \mathrm{MIAA} . \mathbf{i}$, Expression of ER-localized AuxSen (green; first and third rows) and FRET ratio (colour bar; second and fourth rows) of root tissue treated with IAA (top two rows) or DMSO (bottom two rows; control).Scale bar, $50 \mu \mathrm{m}$.j, Quantitative analysis of cells with high (broken lines; IAA, $n=4$; DMSO, $n=4$ ) or low (solid lines; IAA, $n=5$; DMSO, $n=5$ ) levels of AuxSen accumulation. The thick lines represent the mean ( \pm s.d.), the thin lines each represent individual cells. Inset, AuxSen expression in the ER. 


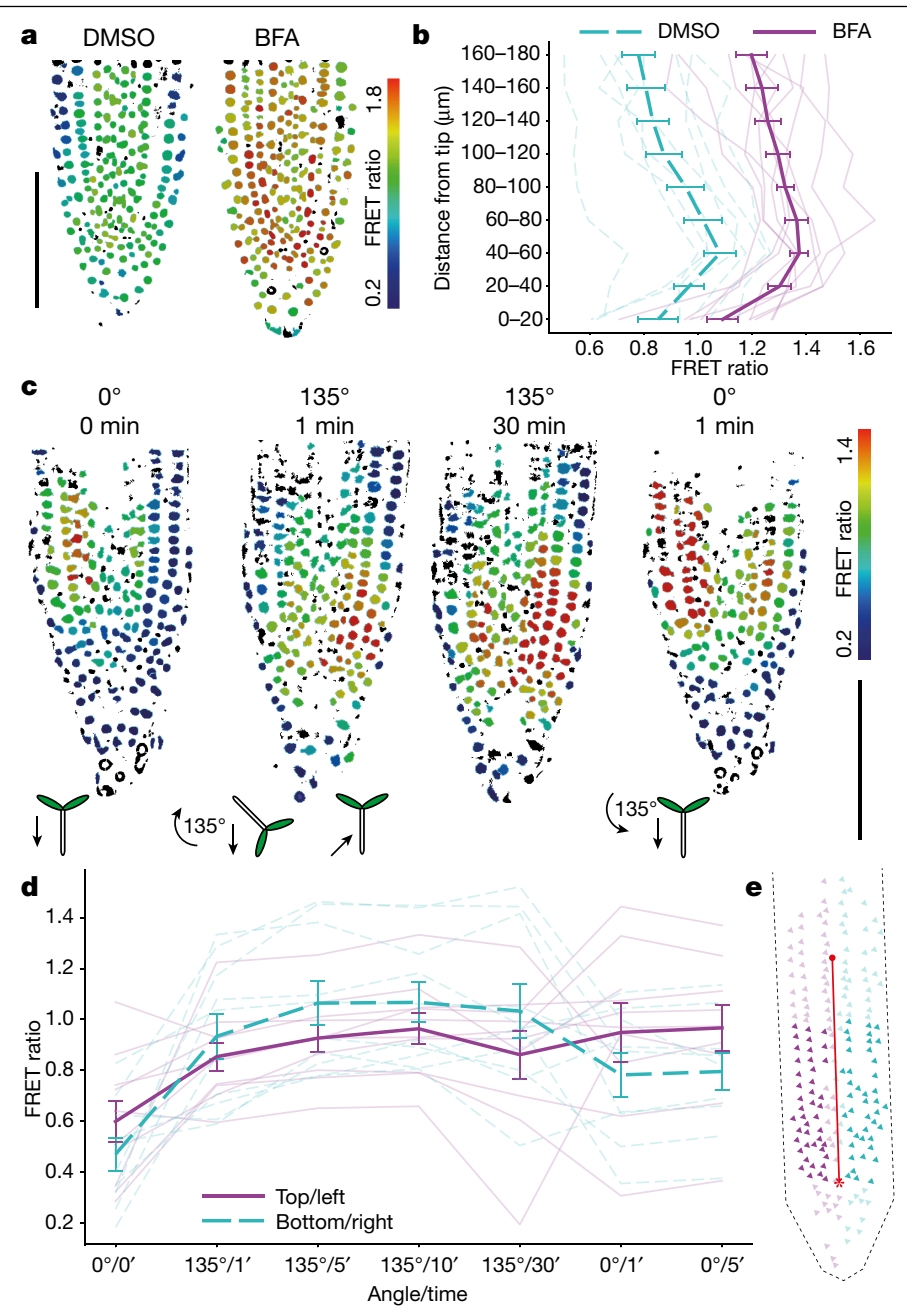

Fig. 4 | FRET ratio of auxin sensor in response to redistribution of endogenous auxin. a, b, Change in the nuclear FRET ratio (colour bar) within the root tip of individual seedlings treated with brefeldin A (BFA) or DMSO. a, Images. b, Quantitative analysis after treatment with BFA $(10 \mu \mathrm{M}$ for $10 \mathrm{~h}$, magenta; $n=10$ ) or DMSO (control, green; $n=9$ ). The thick lines represent the mean ( \pm s.e.m.), the thin lines each represent individual seedlings. c, d, The response of AuxSen to root gravitropism. c, Top, nuclear FRET ratio (colour bar) before and after turning (first and second images), and before and after turning back to the near-vertical position (third and fourth images). Bottom, Cartoons of the seedlings, with arrows indicating the direction of the gravity vector. The signal moves from the left side to the bottom and back to the left side; the colour scale indicates the relative FRET ratio. d, Quantified response of the sensor in individual roots. The thick lines represent the mean ( \pm s.e.m.), the thin lines each represent individual roots $(n=10)$. Scale bars, $100 \mu \mathrm{m}(\mathbf{a}, \mathbf{c})$. e, Diagram of root tip. Nuclei within $100 \mu \mathrm{m}$ above the quiescent centre (asterisk) and at least $10 \mu \mathrm{m}$ from the midline (red line) were analysed (dark triangles). Green, right/bottom; magenta, left/top.

with the solvent DMSO did not induce any response (Fig. 3c, d). To reveal the speed of nuclear auxin accumulation, we measured the FRET signal over shorter time intervals. The maximum signal was reached within 2 min, with more than $80 \%$ achieved after only 1 min (Fig. 3e,f). The uptake of auxin from the extracellular space therefore seems to be highly efficient. We then investigated the reversibility of nuclear accumulation by washing out IAA. After 10 min of incubation of seedlings in $10 \mu \mathrm{M}$ IAA, which gave the maximum FRET ratio, the medium was changed to DMSO (Fig. 3g, h). The FRET ratio gradually decreased, almost reaching the pre-incubation value 30 min later, which suggests slow IAA efflux from the cells (Fig. 3g, h). We repeated the experiment but extended the IAA incubation time to $1 \mathrm{~h}$. This accelerated IAA efflux such that the FRET ratio decreased to its pre-incubation level within 10 min of IAA withdrawal (Fig. 3g, h). This increased efflux of IAA correlated with two- to threefold higher expression levels of PIN efflux carriers after $1 \mathrm{~h}$ compared to 10 min of IAA incubation (Extended Data Fig. 8). In conclusion, this FRET-based sensor can report the dynamics of transient auxin accumulation. Although traditional reporter systems can detect the response to auxin uptake ${ }^{6}$, the irreversibility of reporter translation or degradation obscures the transient nature of the auxin response. Our data suggest that IAA uptake is a constitutive, fast process, whereas the efflux from the cell occurs on demand, as if auxin regulates its own export.

To explore whether auxin might accumulate in other subcellular compartments-those that cannot be accessed by reporters that are based on gene expression or protein degradation-we targeted AuxSen to the lumen of the endoplasmic reticulum(ER). The localization of PIN and PIN-LIKE putative auxin transporters in the ER membrane has led to speculation that auxin accumulation in the ER could be an ancient mechanism of auxin homeostasis ${ }^{23}$. Incubating seedlings in $100 \mu \mathrm{MIAA}$ for $15 \mathrm{~min}$ led to a strong increase in the FRET ratio compared with the DMSO control, suggesting that IAA is transported into the ER (Fig. 3i,j). Different cells within the same seedling root displayed different levels of AuxSen accumulation in the ER (Fig. 3i). Nonetheless, the change in FRET ratio upon exposure to IAA did not differ substantially between cells expressing higher or lower levels of AuxSen (Fig. 3j). In conclusion, this FRET-based auxin sensor can faithfully report auxin concentrations from a subcellular compartment such as the ER, which is inaccessible to traditional auxin-response reporters.

The spatial distribution of endogenous auxin in the seedling root has been inferred from the steady-state expression levels of auxin-response reporters such as $p D R 5: G F P$ or $p 35 S$ :DII-VENUS, which display a pronounced maximum at the quiescent centre of the root meristem near the root tip ${ }^{6}$. Consistent with this, the FRET ratio of our auxin sensor steadily increased towards the root tip, although there was no prominent maximum (Fig. 4a, b). To assess the contribution of transport to the steady-state distribution of auxin, we incubated the seedlings in brefeldin A, which impairs auxin transport by inhibiting the polar recycling of the auxin efflux transporter PIN1 ${ }^{24}$. FRET ratios were increased in the tip of treated roots compared to untreated controls (Fig. 4a,b). This increase presumably resulted from ongoing IAA synthesis in the root tip while efflux was impaired, which is consistent with mass spectrometry analysis of IAA biosynthesis in cell-sorted GFP-expressing lines ${ }^{20}$ and with the expression of auxin-biosynthesis genes in the root tip ${ }^{25}$. We conclude that AuxSen reports the perturbation of endogenous auxin distribution, which highlights its specificity for the detection of auxin.

The redirection of root growth in response to changes in orientation of the gravity vector is a prime example of rapid auxin signalling, which involves redistribution of endogenous auxin within the root tip ${ }^{26}$. Relocalization of the auxin efflux transporter PIN3 from the (former) basal to the (former) lateral plasma membrane has been detected 2 min after turning the root to a horizontal position ${ }^{27}$. However, to our knowledge, no concomitant change in auxin distribution has been reported. The earliest change that has been detected, using the auxin-responsive degradation reporter DII-VENUS, was a reduction in fluorescence in the new lower side 30 min after the onset of gravity stimulation ${ }^{28}$. We equilibrated seedling roots mounted in a near-vertical position for $1 \mathrm{~h}$, then changed their orientation by $135^{\circ}$ and monitored the nuclear FRET ratios over time (Fig. $4 \mathrm{c}$, d). Within $1 \mathrm{~min}$, there was a distinct increase in FRET ratio on the new lower side of the root tip, corresponding to more than $80 \%$ of the maximum value reached after $5 \mathrm{~min}$. We then returned the roots to the near-vertical position after $30 \mathrm{~min}$, and detected substantial recovery of the pre-stimulation distribution of FRET signals within $1 \mathrm{~min}$ (Fig. 4c, d). Gravistimulation therefore elicits a fast and reversible response of the auxin transport system in the root tip. In conclusion, this auxin sensor reports the rapid and reversible redistribution of endogenous auxin accumulation. 


\section{Article}

Our design approach has yielded a new sensor, AuxSen, for the pervasive plant signalling molecule auxin. Starting from a tryptophan sensor, we optimized the affinity and specificity for the binding of IAA, and improved the signal intensity through choice of the FRET pair and optimization of the linkers. Our results provide a proof-of-principle that this detection system can visualize the dynamic redistribution of auxin as well as subcellular pools of auxin, which cannot be achieved with the auxin reporters that are currently in use. An example of insights into auxin dynamics that have been made possible by the resolving power of AuxSen is the contrast between the efficient uptake and the slower, conditional efflux of auxin. Furthermore, AuxSen enables changes in auxin distribution to be distinguished from changes in auxin response, which is a prerequisite for investigating the complex regulatory network that underlies the biological effects of this major signalling molecule in plant growth and development.

\section{Online content}

Any methods, additional references, Nature Research reporting summaries, source data, extended data, supplementary information, acknowledgements, peer review information; details of author contributions and competing interests; and statements of data and code availability are available at https://doi.org/10.1038/s41586-021-03425-2.

1. Enders, T. A. \& Strader, L. C. Auxin activity: Past, present, and future. Am. J. Bot. 102 180-196 (2015)

2. Paque, S. \& Weijers, D. Q\&A: Auxin: the plant molecule that influences almost anything. BMC Biol. 14, 67 (2016)

3. Ulmasov, T., Murfett, J., Hagen, G. \& Guilfoyle, T. J. Aux/IAA proteins repress expression of reporter genes containing natural and highly active synthetic auxin response elements. Plant Cell 9, 1963-1971 (1997).

4. Friml, J. et al. Efflux-dependent auxin gradients establish the apical-basal axis of Arabidopsis. Nature 426, 147-153 (2003).

5. Weijers, D. et al. Auxin triggers transient local signaling for cell specification in Arabidopsis embryogenesis. Dev. Cell 10, 265-270 (2006).

6. Brunoud, G. et al. A novel sensor to map auxin response and distribution at high spatio-temporal resolution. Nature 482, 103-106 (2012)

7. Liao, C. Y. et al. Reporters for sensitive and quantitative measurement of auxin response. Nat. Methods 12, 207-210 (2015)

8. Marmorstein, R. Q., Joachimiak, A., Sprinzl, M. \& Sigler, P. B. The structural basis for the interaction between L-tryptophan and the Escherichia coli trp aporepressor. J. Biol. Chem. 262, 4922-4927 (1987)

9. Retzer, K., Butt, H., Korbei, B. \& Luschnig, C. The far side of auxin signaling: fundamental cellular activities and their contribution to a defined growth response in plants. Protoplasma 251, 731-746 (2014)

10. Leyser, O. Auxin signaling. Plant Physiol. 176, 465-479 (2018)

11. Ma, Q., Grones, P. \& Robert, S. Auxin signaling: a big question to be addressed by small molecules. J. Exp. Bot. 69, 313-328 (2018).
12. Adamowski, M. \& Friml, J. PIN-dependent auxin transport: action, regulation, and evolution. Plant Cell 27, 20-32 (2015).

3. Bennett, T., Hines, G. \& Leyser, O. Canalization: what the flux? Trends Genet. 30, 41-48 (2014).

14. Naramoto, S. Polar transport in plants mediated by membrane transporters: focus on mechanisms of polar auxin transport. Curr. Opin. Plant Biol. 40, 8-14 (2017).

15. Pařízková, B., Pernisová, M. \& Novák, O. What has been seen cannot be unseendetecting auxin in vivo. Int. J. Mol. Sci. 18, 2736 (2017).

16. Tsapakos, M. J., Haydock, P. V., Hermodson, M. \& Somerville, R. L. Ligand-mediated conformational changes in Trp repressor protein of Escherichia coli probed through limited proteolysis and the use of specific antibodies. J. Biol. Chem. 260, 16383-16394 (1985).

17. Zhang, R. G. et al. The crystal structure of trp aporepressor at $1.8 \AA$ A shows how binding tryptophan enhances DNA affinity. Nature 327, 591-597 (1987).

18. Kaper, T. et al. Nanosensor detection of an immunoregulatory tryptophan influx/ kynurenine efflux cycle. PLoS Biol. 5, e257 (2007)

19. Hamers, D., van Voorst Vader, L., Borst, J. W. \& Goedhart, J. Development of FRET biosensors for mammalian and plant systems. Protoplasma 251, 333-347 (2014).

20. Petersson, S. V. et al. An auxin gradient and maximum in the Arabidopsis root apex shown by high-resolution cell-specific analysis of IAA distribution and synthesis. Plant Cell 21, 1659-1668 (2009).

21. Katz, E. et al. The glucosinolate breakdown product indole-3-carbinol acts as an auxin antagonist in roots of Arabidopsis thaliana. Plant J. 82, 547-555 (2015).

22. Herud, O., Weijers, D., Lau, S. \& Jürgens, G. Auxin responsiveness of the MONOPTEROS-BODENLOS module in primary root initiation critically depends on the nuclear import kinetics of the Aux/IAA inhibitor BODENLOS. Plant J. 85, 269-277 (2016).

23. Barbez, E. \& Kleine-Vehn, J. Divide Et Impera-cellular auxin compartmentalization. Curr. Opin. Plant Biol. 16, 78-84 (2013).

24. Geldner, N. et al. The Arabidopsis GNOM ARF-GEF mediates endosomal recycling, auxin transport, and auxin-dependent plant growth. Cell 112, 219-230 (2003)

25. Brumos, J. et al. Local auxin biosynthesis is a key regulator of plant development. Dev. Cell 47, 306-318.e5 (2018).

26. Su, S.-H., Gibbs, N. M., Jancewicz, A. L. \& Masson, P. H. Molecular mechanisms of root gravitropism. Curr. Biol. 27, R964-R972 (2017).

27. Friml, J., Wiśniewska, J., Benková, E., Mendgen, K. \& Palme, K. Lateral relocation of auxin efflux regulator PIN3 mediates tropism in Arabidopsis. Nature 415, 806-809 (2002)

28. Band, L. R. et al. Root gravitropism is regulated by a transient lateral auxin gradient controlled by a tipping-point mechanism. Proc. Natl Acad. Sci. USA 109, 4668-4673 (2012).

Publisher's note Springer Nature remains neutral with regard to jurisdictional claims in published maps and institutional affiliations.

Open Access This article is licensed under a Creative Commons Attribution 4.0 International License, which permits use, sharing, adaptation, distribution and reproduction in any medium or format, as long as you give appropriate credit to the original author(s) and the source, provide a link to the Creative Commons license, and indicate if changes were made. The images or other third party material in this article are included in the article's Creative Commons license, unless indicated otherwise in a credit line to the material. If material is not included in the article's Creative Commons license and your intended use is not permitted by statutory regulation or exceeds the permitted use, you will need to obtain permission directly from the copyright holder. To view a copy of this license visit http://creativecommons.org/licenses/by/4.0/.

(c) The Author(s) 2021 


\section{Methods}

No statistical methods were used to predetermine sample size. The experiments were not randomized and the investigators were not blinded to allocation during experiments and outcome assessment.

\section{Cloning of in-vitro auxin sensors}

The TrpR sensors Trp-CTY and Trp-CTYT were gifts from W. Frommer (Addgene plasmids 13533 and 13534). The fluorophores tested were also donated: Aquamarine by F. Merola (Addgene plasmid 42888), Clover and mRuby 2 by K. Beam (Addgene plasmid 49089), mKO1 by $\mathrm{K}$. Thorn (Addgene plasmid 44642) and mCherry by M. Bayer ${ }^{29}$. eGFP was amplified from pGIIK NLS:3xEGFP ${ }^{30}$. mNeonGreen, mWasabi and mTFP1 were purchased from Allele Biotechnology and Pharmaceuticals, mKate 2 and TagRFP were from Evrogen.

For the initial screening, we first used the Trp-CTY sensor and mutated the residues $\mathrm{T} 44$ and $\mathrm{S} 88$ individually to all possible amino acids. To this end, we generated primers with $15-16$ bp overlaps around the exchanged amino acid codons. We first mutated the amino acids sequence randomly with a degenerate primer and screened 96 clones. Variants not found were then generated by targeted mutagenesis. For example, to generate all T44 variants we first used the primers CCTGATGCTGnnn CCAGATGAGCGCG and CGCGCTCATCTGGnnnCAGCATCAGG, to generate the missing T44C variant we then used the primers CCTGATGCTGtgt CCAGATGAGCGCG and CGCGCTCATCTGGacaCAGCATCAGG. The most promising candidates were introduced by targeted mutagenesis into Trp-CTYT and TrpR without fluorescent proteins, which were analysed by isothermal titration calorimetry (ITC) and structural studies. Trp-CTYT with mutations T44L and S88Y was generated in two steps: we first used the primers CCTGATGCTGctaCCAGATGAGCGCG and CGCGCTCATCTGGtagCAGCATCAGG to generate Trp-CTYT with T44L and then GATTACGCGTGGATCTAACtac-CTGAAAGCCGCGCCC and GGGCGCGGCTTTCAGgtaGTTAGATCCACGCGTAATC to generate Trp-CTYL with the mutations T44L and S88Y. To produce the recombinant proteins, we cloned the TrpR domain into the pET21 expression vector and generated the variants by targeted mutagenesis. This procedure was repeated with the most promising variants after each round. The primer sequences are available upon request.

The final variant was then codon-optimized for Arabidopsis and synthesized by Thermo Fisher Scientific GENEART. To allow an easy exchange of the fluorophores we added restriction enzyme sites at the ends: BamH1 and Xhol around the first fluorophore and Apal and HindIII around the second. All fluorescent proteins were tested in the fluorophore I-TrpR-fluorophore II-TrpR configuration. Having identified Aquamarine and $\mathrm{mNeonGreen}$ as the optimal pair, we introduced all final binding-pocket variants into the backbone by site-directed mutagenesis.

\section{Ligands used for screening and testing}

IAA, TRP, IAN, indole-3-carboxaldehyde, indole, indole-3-acetyl alanine, indole-3-acetyl aspartic acid, indole-3-acetamide, indole-3-ethanol, L-kynurenine, 2-oxindole-3-acetic acid, phenylalanine, picloram, tryptamine, $\left(\mathrm{NH}_{4}\right)_{2} \mathrm{SO}_{4}, \mathrm{CaCl}_{2}, \mathrm{DTT}, \mathrm{H}_{2} \mathrm{O}_{2}, \mathrm{NH}_{4} \mathrm{NO}_{3}, \mathrm{KNO}_{3}$ and yucasin were purchased from Sigma-Aldrich; 4-hydroxyindole-3-carbaldehyde and 5-hydroxyindole-3-carboxylic acid from Santa Cruz Biotechnology; 1-naphthaleneacetic acid, $\mathrm{KCl}$ and DMSO from Carl-Roth; 2,4-dichlorophenoxyacetic acid (2,4-D) from Alfa Aesar; indole-3-acetyl glucose from TRC; indole-3-butyric acid from Serva; $\mathrm{NaCl}$ from Merck; NPA from Supelco; and BFA from Thermo Fisher Scientific.

\section{Mutagenesis}

Trp-CTY variants were generated by site-directed mutagenesis with degenerate or specific oligonucleotides purchased from Sigma-Aldrich. Amplification was carried out using Pfu polymerase (Thermo Fisher Scientific).
More than one thousand oligonucleotides were used; the sequences and resulting vector maps are available upon request. Each variant was sequenced and screened in crude extract of sonicated bacteria for IAA binding; promising candidates were confirmed as purified proteins. The linkers were generated by site-directed mutagenesis, including linkers with $15-16$ bp overlap and 3-9 degenerated nucleotides in the middle. To generate linkers shorter than the original ones, parts were deleted, whereas for longer linkers a fixed sequence was inserted into the middle to reduce the risk of generating stop codons by having too many degenerate nucleotides in the sequence.

\section{Protein expression and purification for screening}

For protein expression, bacteria were grown in the dark on plates with LB-agar supplemented with ampicillin for 3 days at room temperature. To measure crude extracts, we resuspended the bacteria in $20 \mathrm{mM}$ MOPS $\mathrm{pH} 7.2$, sonicated the suspension with an MS 73 probe (Bandelin) and centrifuged the sample with a tabletop centrifuge (Eppendorf). Protein extraction was performed with His Spin trap columns according to the manufacturer's instructions (GE Healthcare). We resuspended the bacteria of one Petri dish in $2 \mathrm{ml}$ binding buffer and sonicated with a MS 73 probe. Buffer exchange was performed with illustra NAP-25 columns (GE Healthcare). All measurements were performed in $20 \mathrm{mM}$ MOPS (Sigma-Aldrich) with an Infinite F200 plate reader (Tecan).

Ligands used for crystallization and ITC. IAA, IAN and TRP used for crystallization and ITC were dissolved in $50 \mathrm{mM} \mathrm{Tris} / 300 \mathrm{mM} \mathrm{NaCl}$ pH 8 buffer containing $1 \%$ DMSO if necessary.

\section{Protein purification for crystallography and ITC}

After subcloning to pET21b(+), wild-type TrpR and all variants were expressed in E. coli BL21(DE3) and purified with a NiIMAC column and a subsequent Superdex-S75 gel-filtration column. All purification steps and measurements were based on the above $50 \mathrm{mM} \mathrm{Tris} / 300 \mathrm{mM} \mathrm{NaCl}$ pH 8 buffer.

\section{Crystallization, data collection and processing}

Crystals of wild-type TrpR and variants with different ligands were obtained by standard vapour diffusion in sitting drop plates. The crystals were cryoprotected if needed and flash-cooled in liquid nitrogen. Data for single crystals were collected at the synchrotron beamline PXII (Swiss Light Source) at $100 \mathrm{~K}$ and 0.5 degree images were recorded on a Pilatus $6 \mathrm{M}$ detector. Only variant TrpR(M42F/T44L/ $\mathrm{T} 81 \mathrm{M} / \mathrm{N} 87 \mathrm{G} / \mathrm{S} 88 \mathrm{Y}$ )-IAA was recorded at MX Beamlines BL14.1 at BESSY II (Helmholtz-Zentrum Berlin für Materialien und Energie). Data were indexed, integrated and scaled with the program XDS and converted with XDSCONV ${ }^{31}$. Molecular replacement was performed with Phenix using the coordinates of wild-type TrpR (PDB ID: $1 W^{2} P^{32}$ or $1 T^{33}{ }^{33}$ ) as search model. Model building was performed using the program $\mathrm{Coot}^{34}$, and refinement was performed using Phenix ${ }^{35}$. Details on crystallization conditions, data and refinement statistics for all structures are summarized in Extended Data Table 2b and Supplementary Table 1.

ITC. ITC was performed using a VP-ITC (MicroCal). The protein concentration was adjusted to $74 \mu \mathrm{M}$ and $730 \mu \mathrm{M}$ ligand solutions were prepared using the above buffer containing $1 \%$ DMSO. Measurements were performed at $20^{\circ} \mathrm{C}$ with a stirring speed of $300 \mathrm{rpm}$, reference power $15 \mu \mathrm{cal} \mathrm{s}^{-1}$ and spacing of $300 \mathrm{~s}$ between injections. The data were analysed using the MicroCal program. Binding data were derived from sigmoidal fits based on a one-site binding model from two measurements for each variant. Heat-of-dilution baselines for the ligands alone were subtracted from the experimental data as previously described ${ }^{36}$. The $\mathrm{pH}$ dependence of IAA binding to the variant $\operatorname{TrpR}(\mathrm{M} 42 \mathrm{~F} / \mathrm{T} 44 \mathrm{~L} /$ $\mathrm{T} 81 \mathrm{M} / \mathrm{N} 87 \mathrm{G} / \mathrm{S} 88 \mathrm{Y}$ ) (AuxSen) was measured on a NanoITC LV device with a stir rate of $300 \mathrm{rpm}, 15$ injections with $2 \mu \mathrm{l}$ and 300 s spacing between injections. The $170 \mu \mathrm{l}$ cell was overfilled with $400 \mu \mathrm{l}$ protein 
to ensure air-free filling. The protein was added at a concentration of $100 \mu \mathrm{M}$ and the ligand IAA at $1 \mathrm{mM}$ at $20^{\circ} \mathrm{C}$. For each $\mathrm{pH}$ the data were recorded in the respective buffer: (i) $50 \mathrm{mM}$ Tris pH $8.5,300 \mathrm{mM} \mathrm{NaCl}$; (ii) $50 \mathrm{mM}$ Tris pH 8.0, $300 \mathrm{mM} \mathrm{NaCl}$; (iii) $50 \mathrm{mM}$ Tris pH 7.5, $300 \mathrm{mM}$ $\mathrm{NaCl}$; (iv) $50 \mathrm{mM}$ Tris pH 7.0, $300 \mathrm{mM} \mathrm{NaCl}$; (v) $50 \mathrm{mM}$ MES pH 6.5, 300 mM NaCl; (vi) 50 mM MES pH 6.0, 300 mM NaCl; (vii) 50 mM MES pH 5.5, 300 mM NaCl; (viii) 50 mM citrate buffer pH 5.0, $300 \mathrm{mM} \mathrm{NaCl}$; and (ix) $50 \mathrm{mM}$ citrate buffer $\mathrm{pH} 4.5,300 \mathrm{mM} \mathrm{NaCl}$.

\section{Test of different FRET pairs}

We tested pairs of Aquamarine, mCerulean3, mTFP1 and mTurquoise2 with Clover, Ypet and $\mathrm{mNeonGreen;} \mathrm{Aquamarine} \mathrm{was} \mathrm{additionally}$ tested with eGFP and mWasabi, and mTFP1 with TagRFP. Furthermore, we tested mNeonGreen, Clover and Ypet with TagRFP and mRuby 2. mKO1 was tested with mCherry, mKate2, mNeonGreen and mWasabi. TagRFP was also tested with mTFP1, mWasabi, mKate2 and mCherry.

\section{Constructs for in-vivo AuxSen experiments}

For protoplast expression, we cloned the final version of the sensor into pJIT60 ${ }^{22}$ (pJIT60-2xp35S:NLS:AuxSen).

For expression in transgenic plants, we cloned the in-vitro optimized AuxSen in constructs for conditional two-component expression, using the pBay-bar vector (a gift from M. Bayer ${ }^{37}$ ). pRPS5a-mGAL4-VP16-GR-UAS_NLS was amplified from a pGII plasmid and inserted into pBay-bar digested with KpnI and BamHI with Gibson Assembly (In-Fusion Cloning, Takara Bio Europe SAS) according to the manufacturer's instructions. In a second step, AuxSen and ocs terminator were inserted into $p B a y$-bar pRPS5a-mGAL4-VP16-GR_UAS_NLS digested with BamHI. To obtain the individual spectra, we replaced AuxSen by meonGreen or Aquamarine. These constructs were used for transforming plants and as a template for the unmix matrix in Fiji. To generate the ER-localized auxin sensor SP:AuxSen:HDEL, we removed the NLS from the nuclear AuxSen construct and inserted the signal peptide of an Arabidopsis vacuolar basic chitinase and the HDEL ER retention sequence ${ }^{38}$ in frame with the coding sequence $\mathrm{N}$-terminally and C-terminally, respectively.

\section{Flow cytometry of protoplasts transiently expressing AuxSen}

Protoplasts were prepared from suspension cell cultures and transfected as previously described ${ }^{39}$, using $12-\mathrm{ml}$ PP tubes and $12 \mu \mathrm{g}$ of construct pJIT60-2xp35S:NLS:AuxSen per $120 \mu \mathrm{l}$ of protoplasts $\left(3.5 \times 10^{6}\right.$ per $\left.\mathrm{ml}\right)$ pertransfection. Onthenext day, transfected protoplasts were pooled, filtered through $100-\mu$ m nylon mesh and split into $200-\mu \mathrm{l}$ aliquots. Each IAA stock (in DMSO) was added 1:100 with a timing offset to account for the 5-min measurement cycle, ensuring a 1-h treatment for each sample, performed in triplicate. Cytometric analysis was set up on a Beckman Coulter MoFlo XDP (100 $\mu \mathrm{m}$ CytoNozzle, 30.5/30.0 psi, PBS sheath) to excite $\mathrm{mNeonGreen} \mathrm{at} 488 \mathrm{~nm}$ (70 mW, elliptical focus) and capture peak FL1 (534/30) and shoulder FL2 (585/29) emission; Aquamarine at $405 \mathrm{~nm}$ excitation $(100 \mathrm{~mW}$, spherical focus) and capture peak FL9 (465/30) and shoulder FL10 (529/28) emission. Data were collected and processed using Summit 5.5 (Beckman Coulter). The main light-scattering gate was determined by identifying the population expressing the greatest amount of reporter. The FRET response was the ratio mean of FL10/FL9, with the auxin response moving towards FL10, directly calculated in Summit 5.5. Representative plots were drawn with FCS Express v.6.06.0033 (deNovo).

\section{Plant material and growth conditions}

Wild-type Arabidopsis thaliana (accession Col-0) plants were used for transformation. Plants were grown on soil at $24{ }^{\circ} \mathrm{C}, 65 \%$ relative humidity under long-day conditions (16-h illumination and 8-h dark period). Seeds were surface-sterilized, stratified for 2 days at $4{ }^{\circ} \mathrm{C}$ and grown on half-strength Murashige and Skoog agar plates containing $1 \%$ sucrose $(0.5 \mathrm{MS}+\mathrm{S})$ (Serva). After 1 week plants were transferred to soil.
For imaging, 4-day-old seedlings were transferred to $0.5 \mathrm{MS}+\mathrm{S}$ $25 \mu \mathrm{M}$ DEX agar plates, and $16 \mathrm{~h}$ later to microscope slides on which they were incubated in $0.5 \mathrm{MS}+\mathrm{S}+\mathrm{IAA}$ or DMSO (control) at the specified concentrations and for the indicated periods of time. To preserve field-of-view and optimal buffer exchange we fixed the cover slip and root with double-sided adhesive tape (Tesa, type 05338, Beiersdorf). To exchange the buffer, we completely emptied the slide on a paper tissue and refilled from the side with the pipette. For BFA treatment, seedlings were transferred to $0.5 \mathrm{MS}+\mathrm{S} 25 \mu \mathrm{M}$ DEX plates containing either $10 \mu \mathrm{MBFA}$ or $0.1 \%$ ( $\mathrm{v} / \mathrm{v}$ ) DMSO (control), and mounted on microscope slides $10 \mathrm{~h}$ later.

\section{Imaging}

The imaging of seedling roots was performed with an LSM780 confocal laser scanning microscope, running ZEN 2.3 black SP1 as acquisition software (Zeiss) and using a Zeiss LD C-Apochromat 40×/1,1 W Korr for all experiments except the gravitropism experiments, which were recorded with a Zeiss Plan-Apochromat $20 \times / 0.8$. Spectral imaging was performed using the QUASAR detection unit on the same system: Aquamarine was excited for FRET ratio measurement at $405 \mathrm{~nm}$ using 5 fluorescent channels $(419-455 \mathrm{~nm}, 454-491 \mathrm{~nm}, 490-526 \mathrm{~nm}$, 525-562 $\mathrm{nm}$ and 561-598 nm); subsequently, mNeonGreen was imaged for segmentation of the regions of interest with excitation at $488 \mathrm{~nm}$ and detection of 3 fluorescent channels (490-526 nm, 525-562 nm and $561-598 \mathrm{~nm}$ ). Gravitropism imaging requiring control over the direction of gravity was performed with a custom-made horizontal imaging kit that can be equipped on most inverted microscope stands for wide-field and confocal imaging. The kit consists of two pieces: a holder for the objective with a mirror for changing the direction of the optical axis of the system from a vertical direction to a horizontal direction (components from Thorlabs). The second piece is a rotatable, vertical sample holder that can be mounted into a standard multiwell plate holder (components from Fischertechnik).

We used spectral $\mathrm{FRET}^{40}$ to be able to control for influences from sources of autofluorescence, which can be abundant in plant tissues ${ }^{41}$. Spectral FRET therefore also has the advantage that the method can be adapted by adjusting the number of acquisition channels if other sources of autofluorescence are present in different plant tissues.

All analyses were performed using a current version of $\mathrm{Fiji}^{42}$. First, signals generated were linearly unmixed ${ }^{43}$ using J. Walter's spectral unmixing plugin (https://imagej.nih.gov/ij/plugins/spectral-unmixing. html). The unmixing matrix was generated with mNeonGreen, and Aquamarine as fluorophore controls and a wild-type Col-0 control for background autofluorescence. After this, the images were manually thresholded on all channels to remove unspecific signals and saturated areas, regions of interest (ROIs) for the cell nuclei were automatically generated based on the $488 \mathrm{~nm} / 490-526 \mathrm{~nm}$-channel data using an adaptive threshold plugin (by Q. Tseng, https://sites.google.com/site/ qingzongtseng/adaptivethreshold) and the 'Watershed' and 'Analyze Particles' functions of ImageJ. We analysed all pixels of the image only for the ER. The FRET ratio was calculated by spectral unmixing of the channels using 'Spectral Unmix' version 1.3 (by J. Walter, https://imagej. nih.gov/ij/plugins/spectral-unmixing.html) and a precomputed unmixing matrix (see above) yielding the Aquamarine and mNeonGreen emission for division. Unmixed ROIs were colour-coded using the 'ROI Color Coder' plugin (BAR library, by T. Ferreira, http://imagejdocu.tudor.lu/ doku.php?id=macro:roi_color_coder). In general, the colour scales were adapted for each experiment best reflecting the differences. Nuclei consisting of areas that were too small or those that had unrealistic high FRET ratios (owing to insufficient Aquamarine signal) were omitted (reflected as black in the colour coding).

\section{Reporting summary}

Further information on research design is available in the Nature Research Reporting Summary linked to this paper. 


\section{Data availability}

All processed data generated or analysed during this study are included in this Article and its Supplementary Information. Imaging (Herud_et_al_2021_figures_3_and_4_raw_data) and in vitro source data (Herud_et_al_2021_suppl_figures_S1_S3-S5_S7_and_table_S2a_raw_data) are available at Zenodo (https://doi.org/10.5281/zenodo.4524537). The data for the FACS measurements in Fig. 3a, b and Extended Data Figs. 6, 7 can be found at https://flowrepository.org (FR-FCM-Z3FL). Coordinates and structure factors for all reported X-ray crystallography structures have been deposited in the PDB under accession codes 6EJW, 6EJZ, 6ENI, 6EKP, 6ENN, 6ELB, 6ELF and 6ELG. Materials (transgenic lines and plasmids) will be made available via the Arabidopsis Biological Resource Center (ABRC) in Columbus ( $\mathrm{OH}, \mathrm{USA})$ and the Nottingham Arabidopsis Stock Centre (NASC), Nottingham (UK).

\section{Code availability}

The ImageJ macros used to analyse data (Herud_et_al_2021_ImageJ_ analysis_macros) are available from Zenodo (https://doi.org/10.5281/ zenodo.4524537).

29. Musielak, T. J., Slane, D., Liebig, C. \& Bayer, M. A versatile optical clearing protocol for deep tissue imaging of fluorescent proteins in Arabidopsis thaliana. PLOS ONE 11 e0161107 (2016)

30. Takada, S. \& Jürgens, G. Transcriptional regulation of epidermal cell fate in the Arabidopsis embryo. Development 134, 1141-1150 (2007).

31. Kabsch, W. XDS. Acta Crystallogr. D 66, 125-132 (2010).

32. Lawson, C. L. et al. Flexibility of the DNA-binding domains of trp repressor. Proteins $\mathbf{3}$, 18-31 (1988).

33. Otwinowski, Z. et al. Crystal structure of trp repressor/operator complex at atomic resolution. Nature 335, 321-329 (1988).

34. Emsley, P. \& Cowtan, K. Coot: model-building tools for molecular graphics. Acta Crystallogr. D 60, 2126-2132 (2004).

35. Adams, P. D. et al. PHENIX: a comprehensive Python-based system for macromolecular structure solution. Acta Crystallogr. D 66, 213-221 (2010).

36. Machius, M. et al. Structural and biochemical basis for polyamine binding to the Tp0655 lipoprotein of Treponema pallidum: putative role for Tp0655 (TpPotD) as a polyamine receptor. J. Mol. Biol. 373, 681-694 (2007)

37. Wang, K. et al. Independent parental contributions initiate zygote polarization in Arabidopsis thaliana. Preprint at https://doi.org/10.1101/2020.12.02.407874 (2020)

38. Haseloff, J., Siemering, K. R., Prasher, D. C. \& Hodge, S. Removal of a cryptic intron and subcellular localization of green fluorescent protein are required to mark transgenic Arabidopsis plants brightly. Proc. Natl Acad. Sci. USA 94, 2122-2127 (1997).

39. Mehlhorn, D. G., Wallmeroth, N., Berendzen, K. W. \& Grefen, C. 2in1 vectors improve in planta BiFC and FRET analyses. Methods Mol. Biol. 1691, 139-158 (2018).

40. Piston, D. W. \& Kremers, G. J. Fluorescent protein FRET: the good, the bad and the ugly. Trends Biochem. Sci. 32, 407-414 (2007).
41. Donaldson, L. Autofluorescence in plants. Molecules 25, 2393 (2020).

42. Schindelin, J. et al. Fiji: an open-source platform for biological-image analysis. Nat. Methods 9, 676-682 (2012)

43. Neher, R. \& Neher, E. Optimizing imaging parameters for the separation of multiple labels in a fluorescence image. J. Microsc. 213, 46-62 (2004).

44. Lambert, T. J. FPbase: a community-editable fluorescent protein database. Nat. Methods 16, $277-278$ (2019)

45. Novák, O. et al. Tissue-specific profiling of the Arabidopsis thaliana auxin metabolome. Plant J. 72, 523-536 (2012).

46. Tam, Y. Y., Epstein, E. \& Normanly, J. Characterization of auxin conjugates in Arabidopsis Low steady-state levels of indole-3-acetyl-aspartate, indole-3-acetyl-glutamate, and indole-3-acetyl-glucose. Plant Physiol. 123, 589-596 (2000)

47. Mashiguchi, K. et al. The main auxin biosynthesis pathway in Arabidopsis. Proc. Natl Acad. Sci. USA 108, 18512-18517 (2011).

48. Normanly, J., Cohen, J. D. \& Fink, G. R. Arabidopsis thaliana auxotrophs reveal a tryptophan-independent biosynthetic pathway for indole-3-acetic acid. Proc. Natl Acad. Sci. USA 90, 10355-10359 (1993).

49. Böttcher, C. et al. The biosynthetic pathway of indole-3-carbaldehyde and indole3-carboxylic acid derivatives in Arabidopsis. Plant Physiol. 165, 841-853 (2014).

Acknowledgements We thank Addgene for distributing plasmids donated by W. Frommer F. Merola, K. Beam and K. Thorn; O. Griesbeck, H. Schäfer, A. Holz, A.-C. Barragan-Lopez and C. Brancato for technical assistance; M. Perez Ortega for the initial steps towards the macro script; M. Belkacemi for sequencing of the variants; and M. Bayer for discussions and suggestions. C.F. thanks R. Palmisano and M. Küppers for help with gravitopism kit design. O.H.-S., M.K. and C.F. thank the staff of the Light Microscopy Facility at MPI for Developmental Biology for their support. G.J. thanks R. Hertel for discussions on auxin-related matters. We further thank the beamline staff at the Swiss Light Source and at BESSY for support. B.H. acknowledges the financial support and the allocation of synchrotron beam time by HZB as well as funding by the Deutsche Forschungsgemeinschaft (grant $\mathrm{HO} 4022 / 2-3$ ). This study was supported by the Max Planck Society.

Author contributions O.H.-S., A.C.S., B.H. and G.J. conceived the idea and designed the experiments. O.H.-S., A.C.S., M.K., S.S. and K.W.B. performed the experiments. O.H.-S. performed cloning and mutagenesis of auxin sensor constructs for in vitro analysis, A.C.S. and S.S. carried out biochemistry and structure analysis of auxin sensor variants, and O.H.-S., A.C.S. and S.S. performed ligand-binding studies. M.K. cloned the auxin sensor constructs for in-vivo analysis, generated transgenic lines, analysed AuxSen expression, performed microscopic imaging analyses and determined auxin transporter expression. K.W.B. performed the transfection and flow cytometry analysis of the auxin sensor in protoplasts. C.F. developed the imaging workflow and constructed the microscope adapter for gravitropism-related measurements. O.H.-S., A.C.S., B.H. and G.J. wrote the manuscript with input from all authors.

Funding Open access funding provided by Max Planck Society.

Competing interests The authors declare no competing interests.

Additional information

Supplementary information The online version contains supplementary material available at https://doi.org/10.1038/s41586-021-03425-2.

Correspondence and requests for materials should be addressed to B.H. or G.J.

Peer review information Nature thanks Richard Napier and the other, anonymous, reviewer(s) for their contribution to the peer review of this work.

Reprints and permissions information is available at http://www.nature.com/reprints. 

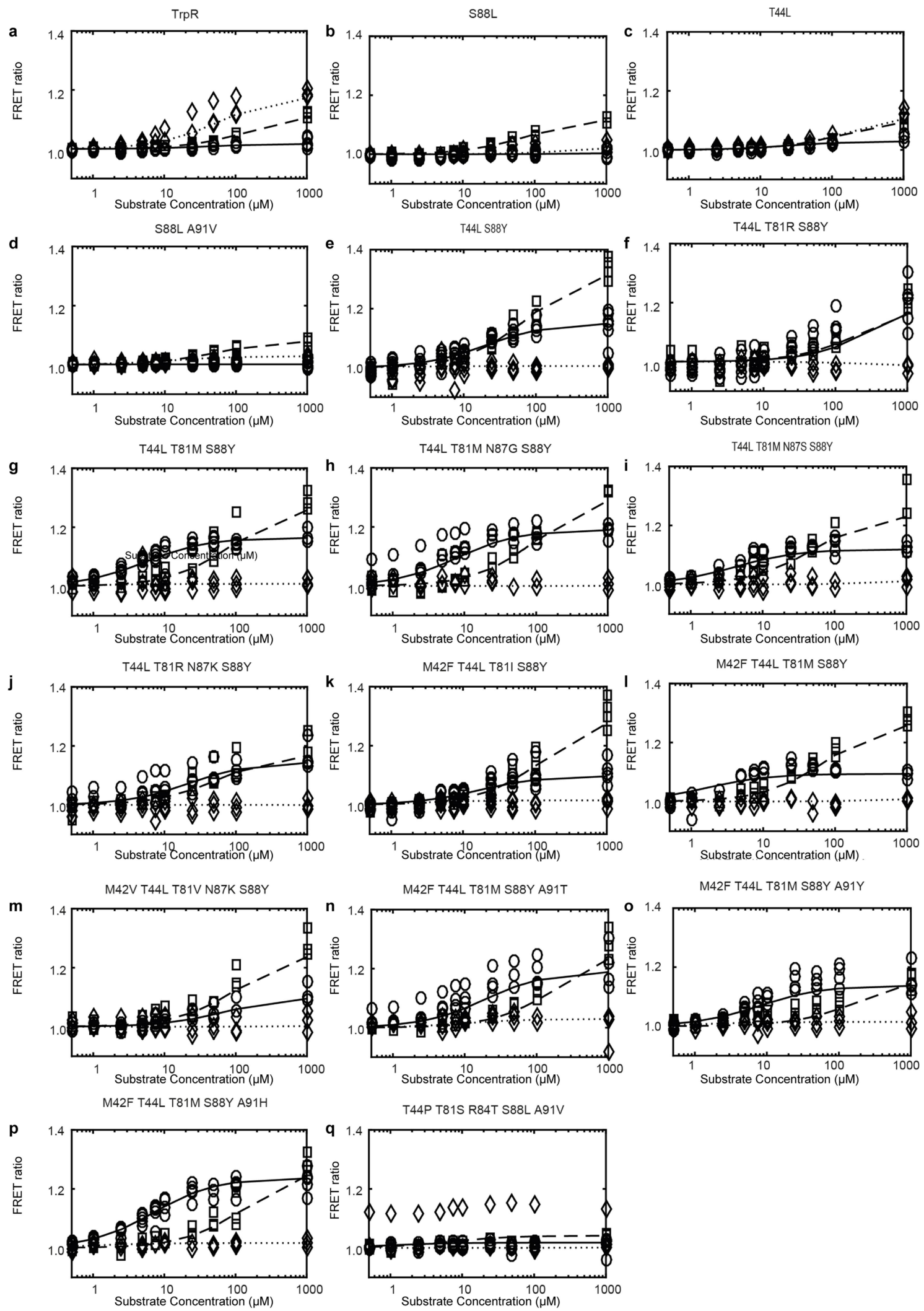

Extended Data Fig. 1 | FRET response of several binding-domain variants to increasing substrate concentration. a, Wild-type TrpR. b-q, Engineered TrpR variants. Each mark indicates a single measurement. Circle, IAA; rhombus, TRP; square, IAN. 


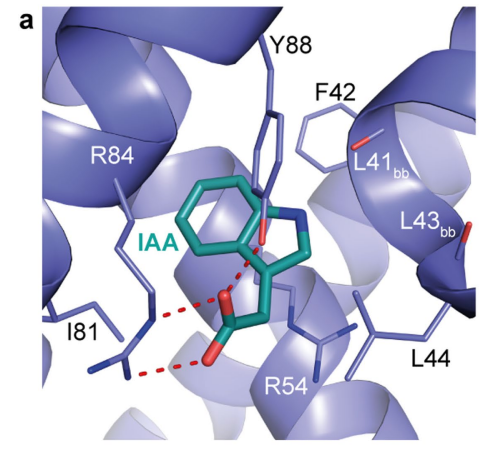

e

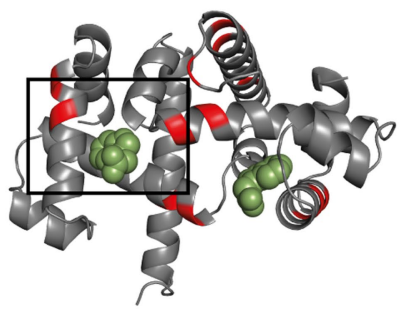

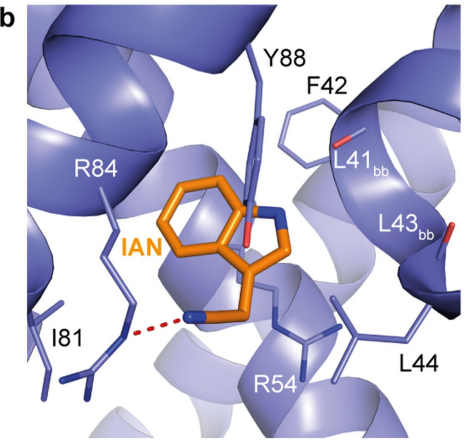

f

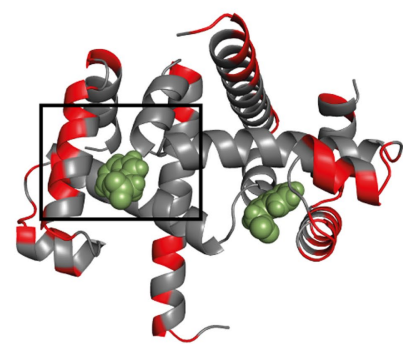

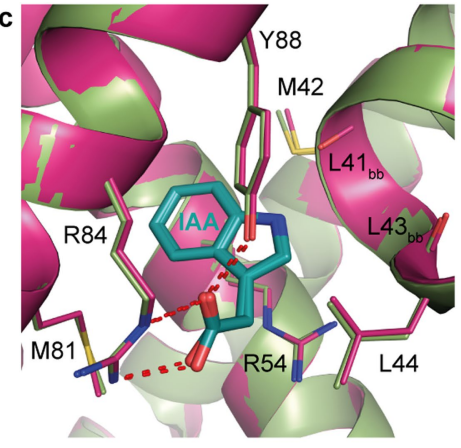

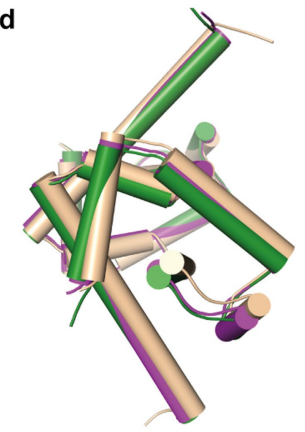

g

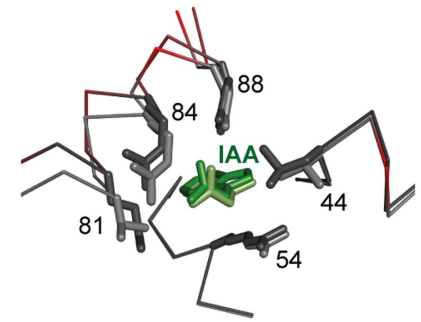

Extended Data Fig. 2 | Details observed in the crystal structures.

a, Structure of IAA in the binding pocket of $\operatorname{TrpR}(\mathrm{M} 42 \mathrm{~F} / \mathrm{T} 44 \mathrm{~L} / \mathrm{T} 81 \mathrm{I} / \mathrm{S} 88 \mathrm{Y})$. b, Structure of IAN bound to the same variant as in a.c, Overlay of IAA in $\operatorname{TrpR}(\mathrm{T} 44 \mathrm{~L} / \mathrm{T} 81 \mathrm{M} / \mathrm{S} 88 \mathrm{Y}$ ) (magenta) and $\operatorname{TrpR}(\mathrm{T} 44 \mathrm{~L} / \mathrm{T} 81 \mathrm{M} / \mathrm{N} 87 \mathrm{G} / \mathrm{S} 88 \mathrm{Y}$ ) (green). d, Structural overview of variants $\operatorname{TrpR}(\mathrm{T} 44 \mathrm{~L} / \mathrm{T} 81 \mathrm{M} / \mathrm{N} 87 \mathrm{G} / \mathrm{S} 88 \mathrm{Y})$ (green), $\operatorname{TrpR}(\mathrm{T} 44 \mathrm{~L} / \mathrm{T} 81 \mathrm{M} / \mathrm{S} 88 \mathrm{Y})$ (magenta), and TrpR(M42F/T44L/T81M/N87G/S88Y) (AuxSen, gold). It is apparent that AuxSen differs from the two intermediate structures regarding the overall arrangement of the helices. $\mathbf{e}-\mathbf{g}$, The structure of $\operatorname{TrpR}(\mathrm{S} 88 \mathrm{Y} / \mathrm{T} 44 \mathrm{~L})$ and all variant structures based on it show a slight relocation of the backbone of residues 70-90. This is probably due to the fact that all structures based on this variant crystallize in the orthorhombic space group $P 2_{1} 2_{1} 2_{1}(f)$ as opposed to the tetragonal space group $P 4_{3}$ found for TrpR-IAA and TrpR(S88Y)-IAA (e). Both geometries have been found in earlier crystal structures of TrpR, for example PDB 1ZT9 (tetragonal) and $20 Z 9$ (orthorhombic). It seems that the introduction of the T44L mutation strongly favours crystallization in the orthorhombic geometry. In the $P 2_{1} 2_{1} 2_{1}$ space group, crystals form more extensive crystal contacts. The structure overlay (g) shows how several residues are displaced (residues that have symmetry mates within $3 \AA$ are shown in red, and the ligand IAA is shown in green). However, interactions and positioning of the ligands are maintained. Nonetheless, we only compare backbone coordinates between variants of the same space group, to exclude misinterpretations due to crystal contacts. 
a

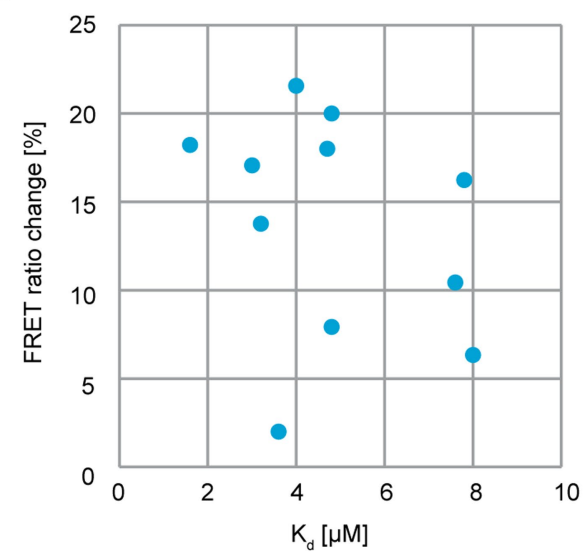

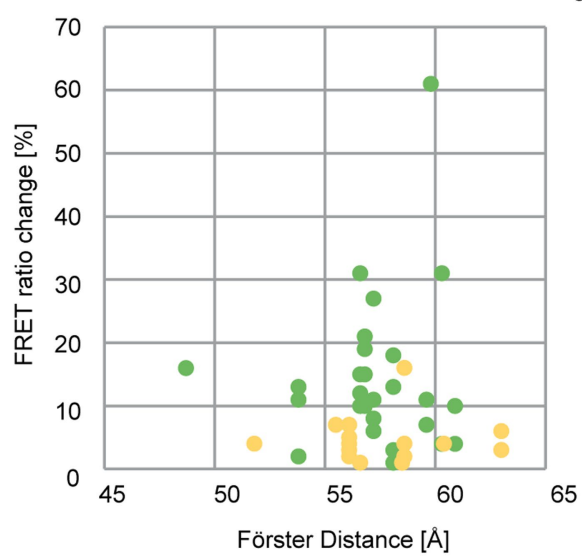

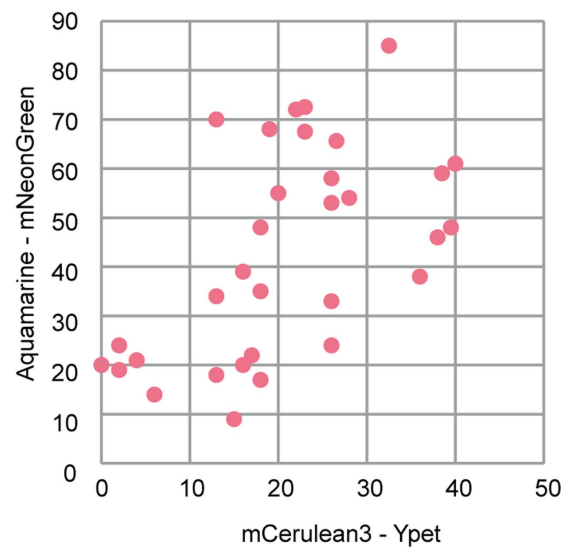

d

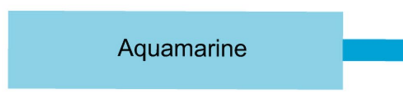

e

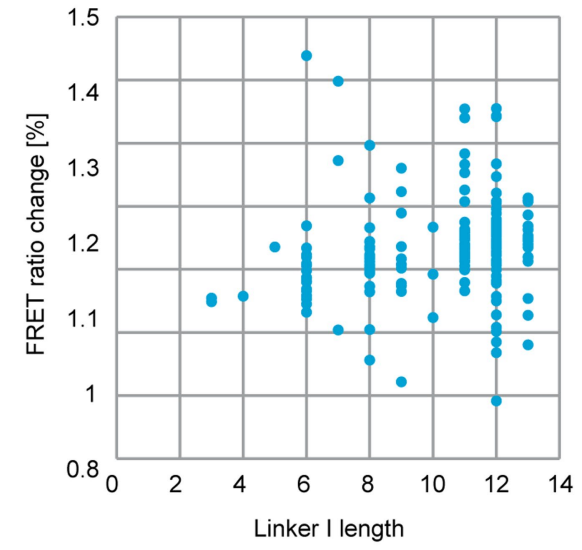

$\mathrm{h}$

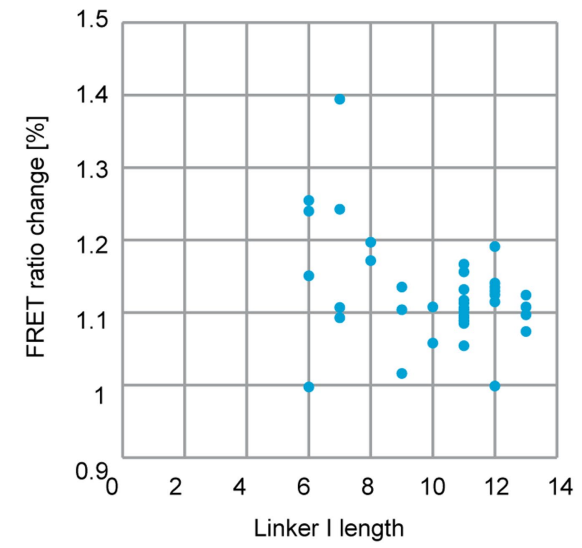

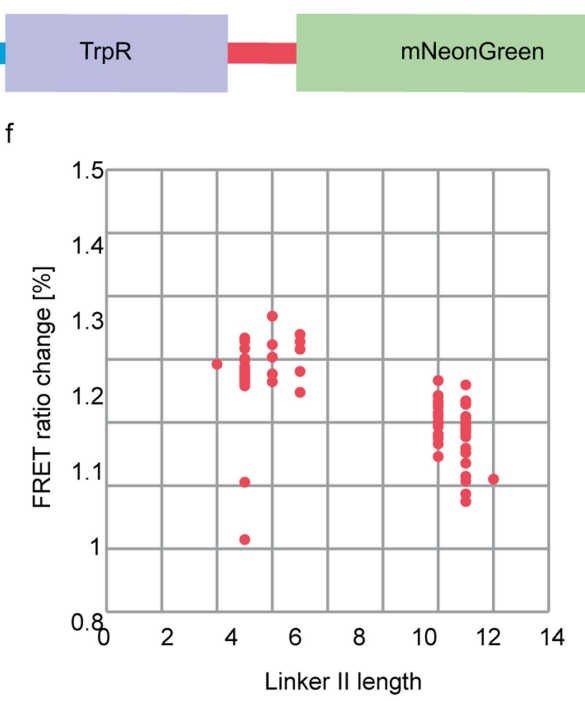

i

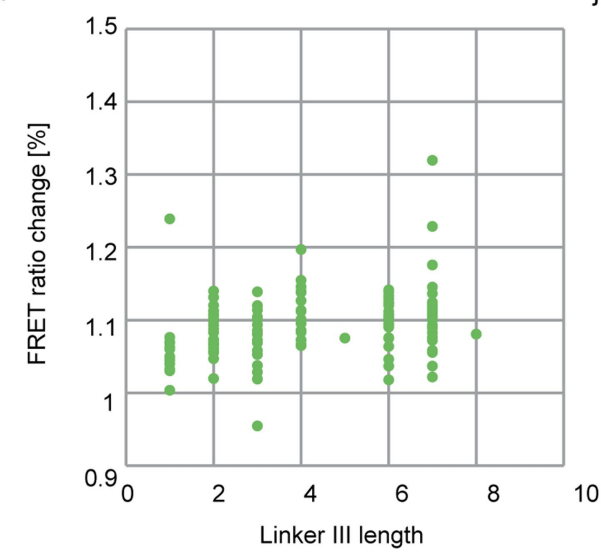

g

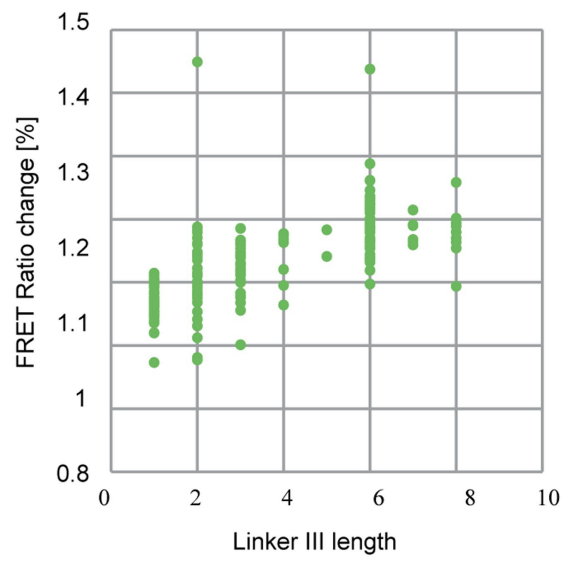

j

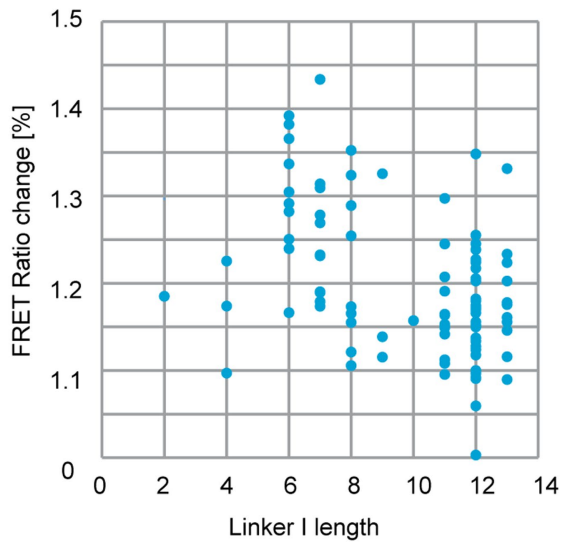

Extended Data Fig. 3 | Parameters tested for potential influence on the change of FRET ratio. a, Change in FRET ratio upon IAA treatment plotted against the dissociation constant $\left(K_{\mathrm{d}}\right)$ of the same variant as determined by ITC. b, FRET ratio changes do not correlate with the Förster distance. Blue-yellow pairs are marked in green, yellow-red pairs are in orange. Blue-yellow pairs, in general, show a higher FRET ratio change upon IAA treatment, but a similar range of Förster distances as the yellow-red ones. c, FRET ratio changes (in per cent) of several variants tested with two different fluorophore pairs. Variants showing a strong response with one fluorophore pair usually also show a strong response with another pair (correlation coefficient $=0.6$ ). $\mathbf{d}-\mathbf{j}$, Effects of mutations in linkers. d, Structure of the construct. The IAA-binding TrpR variants were cloned as tandem repeats into the construct containing donor and acceptor fluorophores, analogous to ref. ${ }^{18}$. The positional effect of the fluorescent proteins probably stems from slight rearrangements of the overall backbone in both TrpR subunits. Predominantly, this involves helix E of the reading-head motif, which mediates the DNA interaction in the natural function of TrpR. Because helix E is towards the C-terminal end of the chain, is it thought that fluorescent proteins positioned at this end will experience a larger positional relocation and thus show a more dynamic range of the FRET signal. e-g, First-round linker mutations. All three linkers were mutated, but no pattern for the optimal linker length could be determined. One linker II variant was chosen for further mutations. $\mathbf{h}, \mathbf{i}$, Second-round linker mutations. Linkers I and III were mutated in the variant obtained in the first round, with no changes in the optimized linker II.j, Third-round linker mutations. Linker I was further mutated in the variant containing mutations in linkers II and III. The linker length axis indicates the number of amino acid residues. 
a

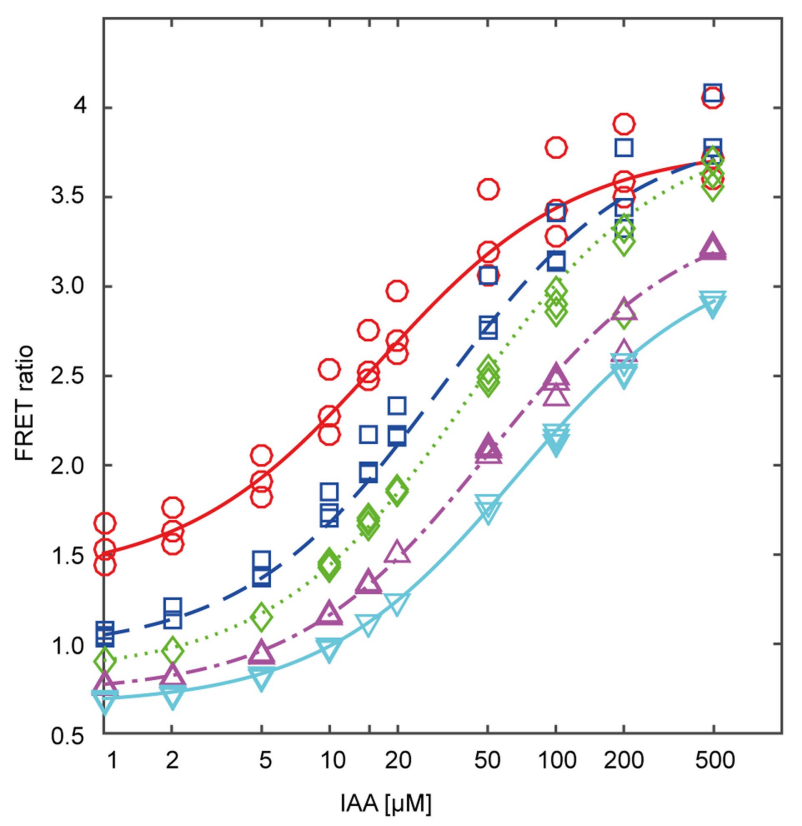

C

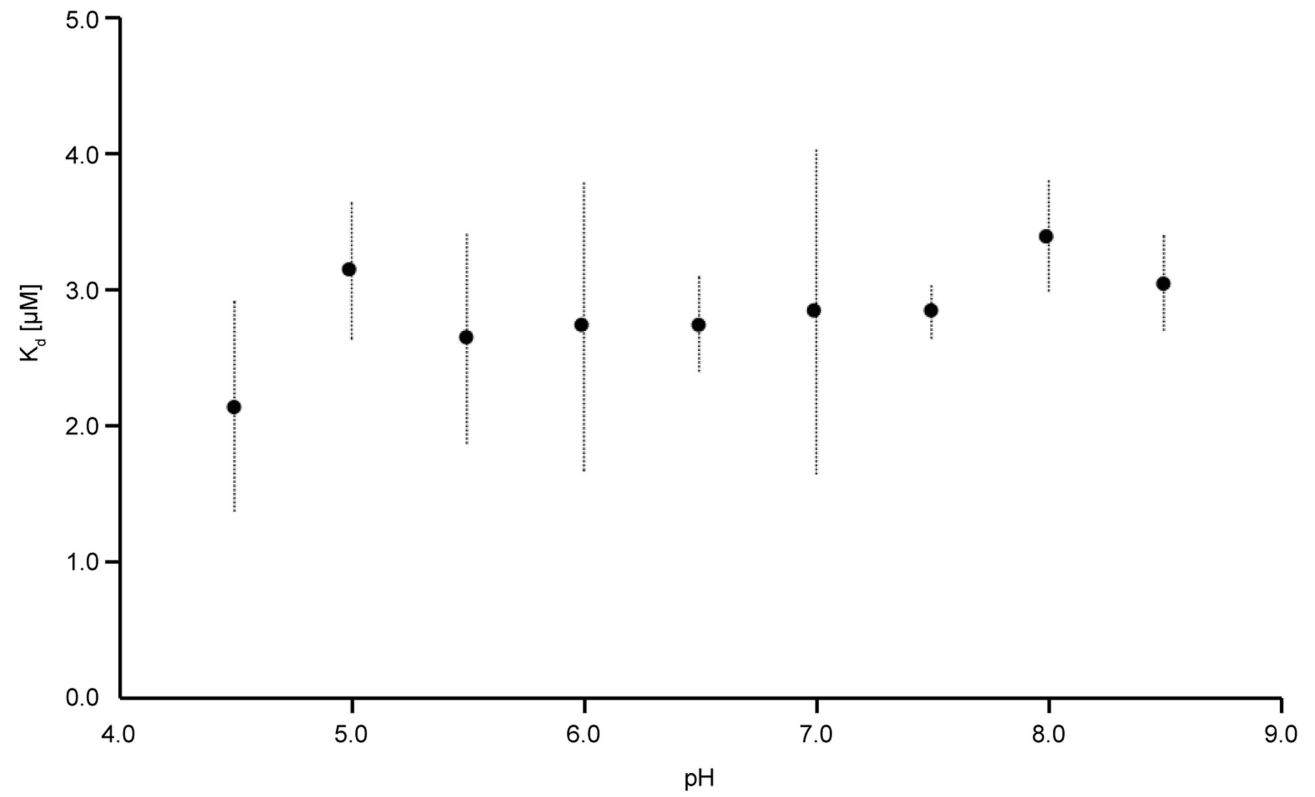

Extended Data Fig. 4 | pH, salt and redox sensitivity of AuxSen. a, The FRET ratio is slightly affected by changes in the $\mathrm{pH}$, but fully functional in the range of $\mathrm{pH}$ values within the plant cell. $\mathrm{Red}, \mathrm{pH} 6.0$; blue, $\mathrm{pH}$ 6.5; green, $\mathrm{pH}$ 7.0; violet, pH 7.5; cyan, $\mathrm{pH}$ 8.0. b. The FRET ratio is not strongly affected by salts and changes in the redox potential. Black, control; red, $1 \mathrm{mM}\left(\mathrm{NH}_{4}\right)_{2} \mathrm{SO}_{4}$; blue,

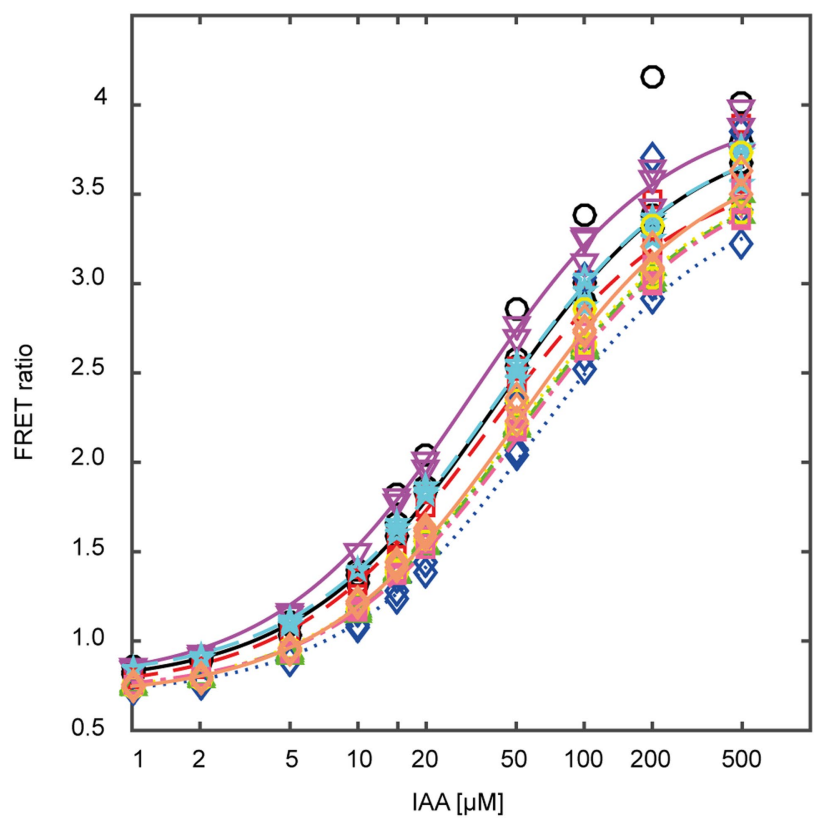

$1 \mathrm{mM} \mathrm{CaCl}_{2}$; green, $10 \mathrm{mM} \mathrm{NH}_{4} \mathrm{NO}_{3}$; violet, $10 \mathrm{mMDTT}$; cyan, $10 \mathrm{mM} \mathrm{H}_{2} \mathrm{O}_{2}$; yellow, $10 \mathrm{mM} \mathrm{KCl}$; orange squares, $10 \mathrm{mM} \mathrm{KNO}_{3}$; orange diamonds, $10 \mathrm{mM}$ $\mathrm{NaCl}$. c, ITC data of AuxSen binding to IAA, measured at different $\mathrm{pH}$ values. Data are mean \pm s.d., derived from 3 technical replicates. 


\section{Article}
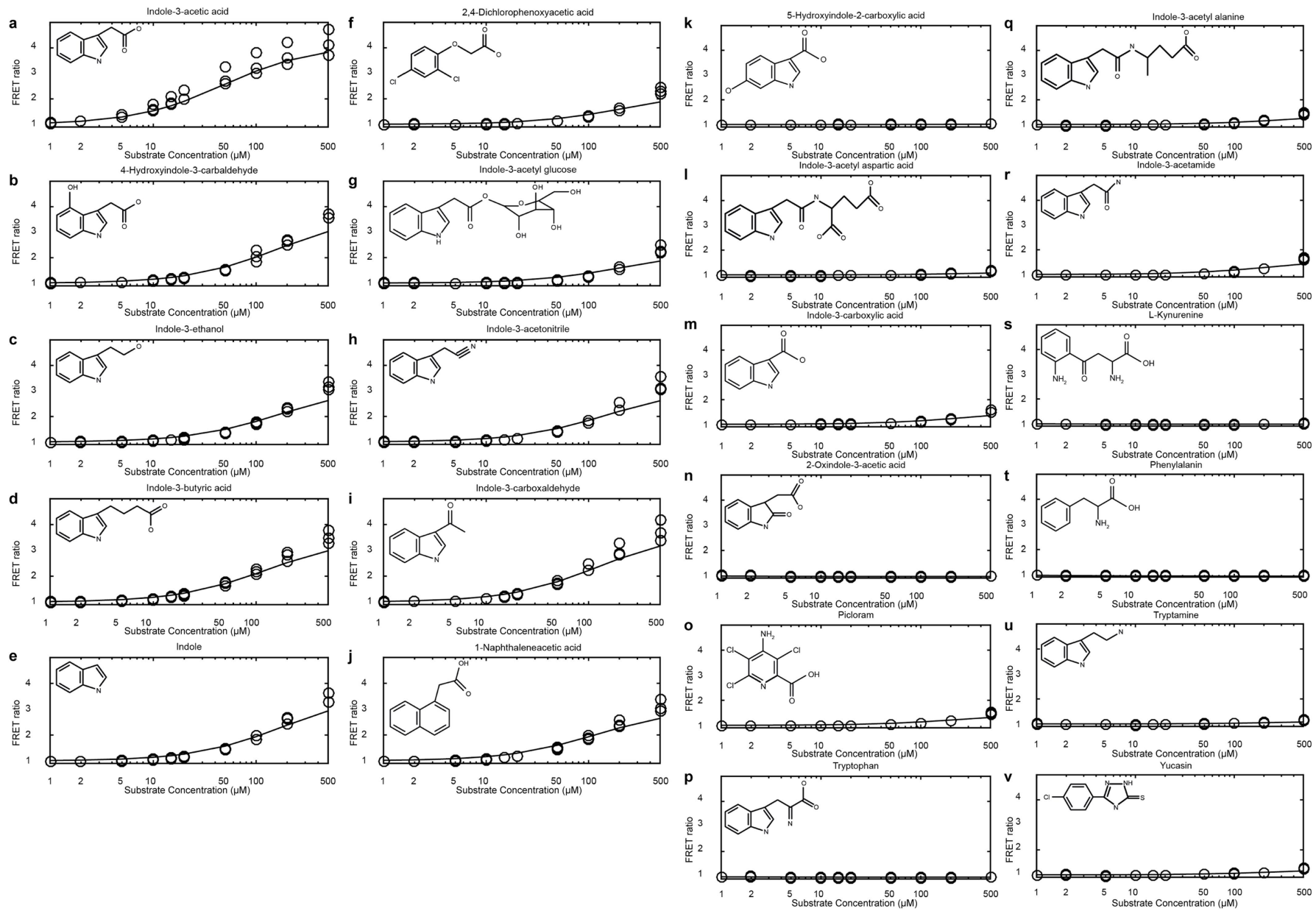

Extended Data Fig. $\mathbf{5}$ | Affinities of AuxSen for auxin-related compounds. a-j, Compounds with weak affinities; $\mathbf{k}-\mathbf{v}$, compounds with no affinity. The change in FRET ratio $(y$ axis) is plotted against increasing concentrations of the individual compounds in $\mu \mathrm{M}(x$ axis). Each mark indicates a single measurement. 
a

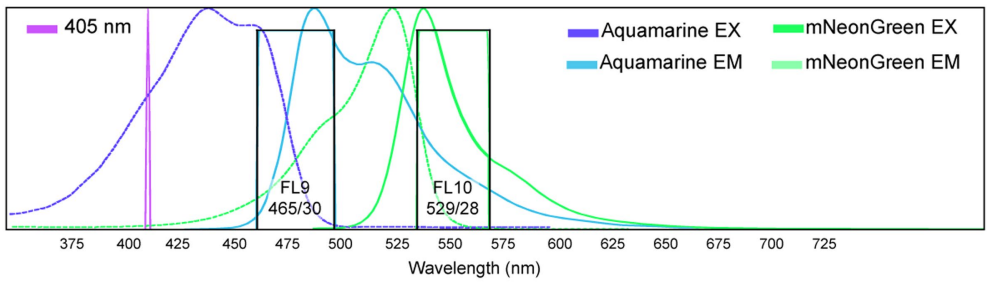

b

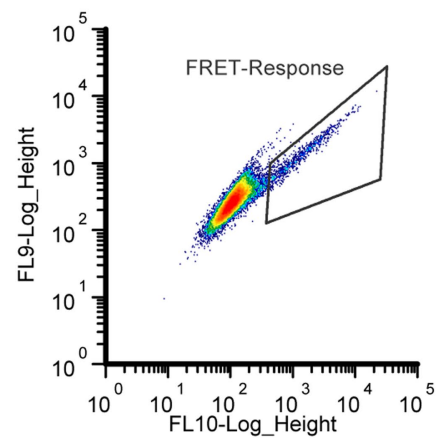

Extended Data Fig. 6 | FRET ratio of AuxSen quantified in protoplasts. Cell-suspension-culture protoplasts were transfected with $p / I T 6 O$ 2xp35SS:NLS:AuxSen and the FRET response was captured by flow cytometry as the ratio of the emission between the Aquamarine peak intensity (FL9) and mNeonGreen peak intensity (FL10) as 'Log_Height' (a.u.). a, Normalized c

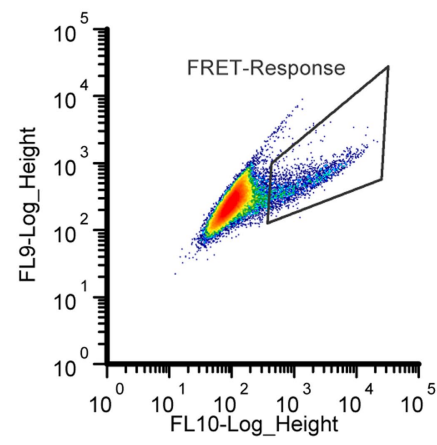

excitation and emission spectra for mNeonGreen and Aquamarine. The 405-nm laser line is shown as a vertical bar and bandpass filters are shown as open boxes, as rendered by FPBase ${ }^{44}$. As FRET occurs, the emission output is shifted from the FL9 to the FL10 bandpass. b, Baseline level of AuxSen FRET response, without exogenous IAA. c, Maximal response level of AuxSen with 10 mM IAA. 

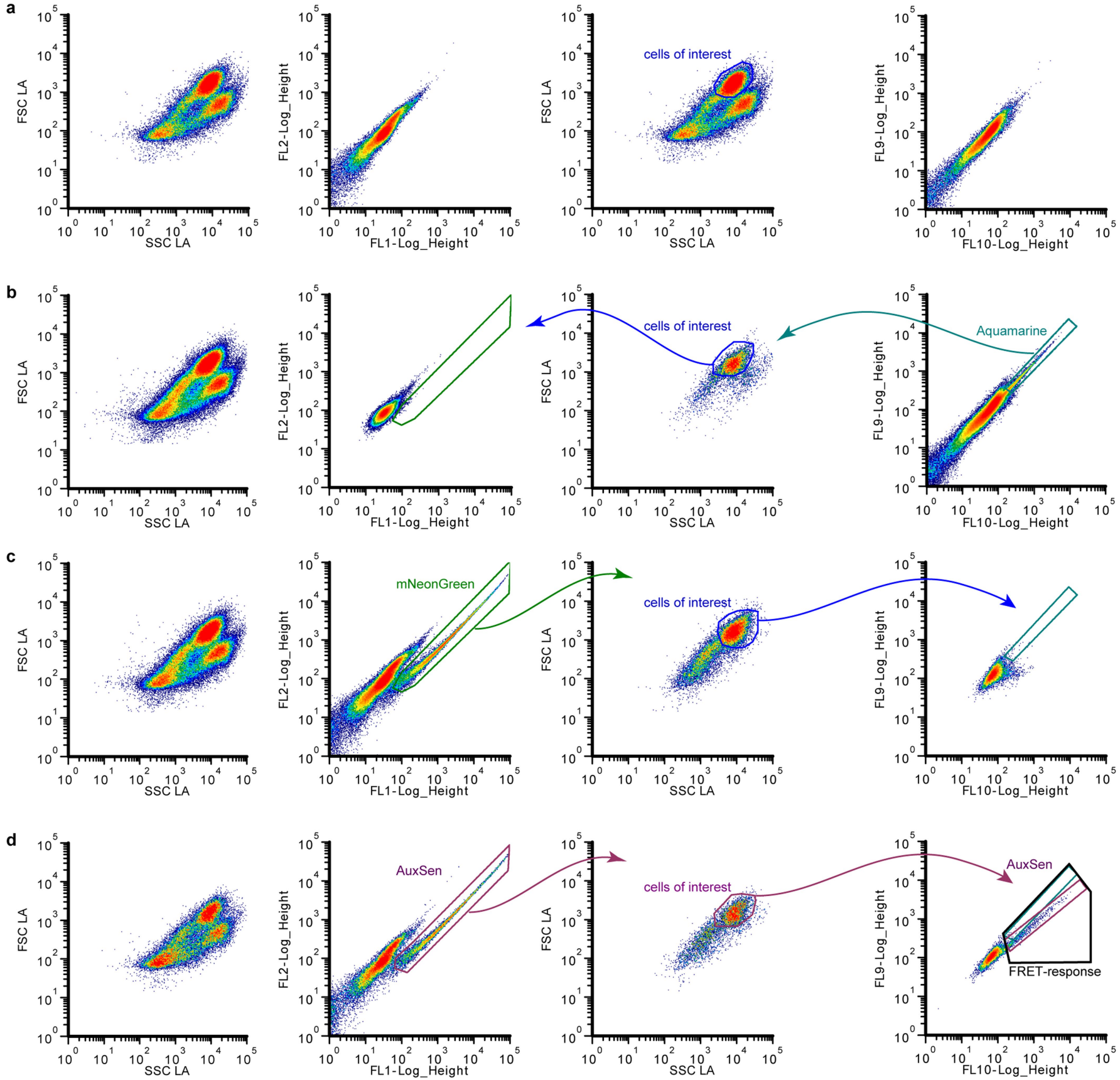

Extended Data Fig. $7 \mid$ Demonstration of relevant population regions and Aquamarine, mNeonGreen and AuxSen fluorescent emission in cell-suspension-culture protoplasts. Bivariate plots from left to right are as follows: forward versus side-scatter log area ungated; emission peak to shoulder FL1 (534/30) versus FL2 (585/29); forward versus side-scatter log area with cells of interest marked and gated for $\mathbf{b}-\mathbf{d}$; emission peak-to-shoulder FL9 $(465 / 30)$ versus FL10 (529/28). Arrows indicate 'gating', meaning that the following plot is restricted to those data points that fall within that particular window. a, Cells only transfected with water.b. Cells transfected with $p J I T 6 O$ 2xp35SS:NLS:Aquamarine. Cells expressing Aquamarine were used to determine which scattering population produced the fluorescent protein. This gate is followed for mNeonGreen emission. c, Cells transfected with $p J I T 6 O$ -

2xp35SS:NLS:mNeonGreen. Cells expressing mNeonGreen were used to determine which scattering population produced the fluorescent protein. This gate is followed for Aquamarine emission. d, Cells transfected with $p J I T 6 O$ 2xp35SS:NLS:AuxSen. Cells expressing AuxSen identified by their mNeonGreen emission then restricted to the 'cells of interest' scattering population that produced the greatest amount of protein. The FRET-response region was then made to encompass the entire range of possible fluorescence, including the shift in the FL9 to the FL10 bandpasses. See also Supplementary Table 2. Flow cytometry basic gate statistics and FRET-ratios based on ('FL10-Log Height'/'FL9-Log_Height') ratio are plotted against time for the final gate FRET response. 


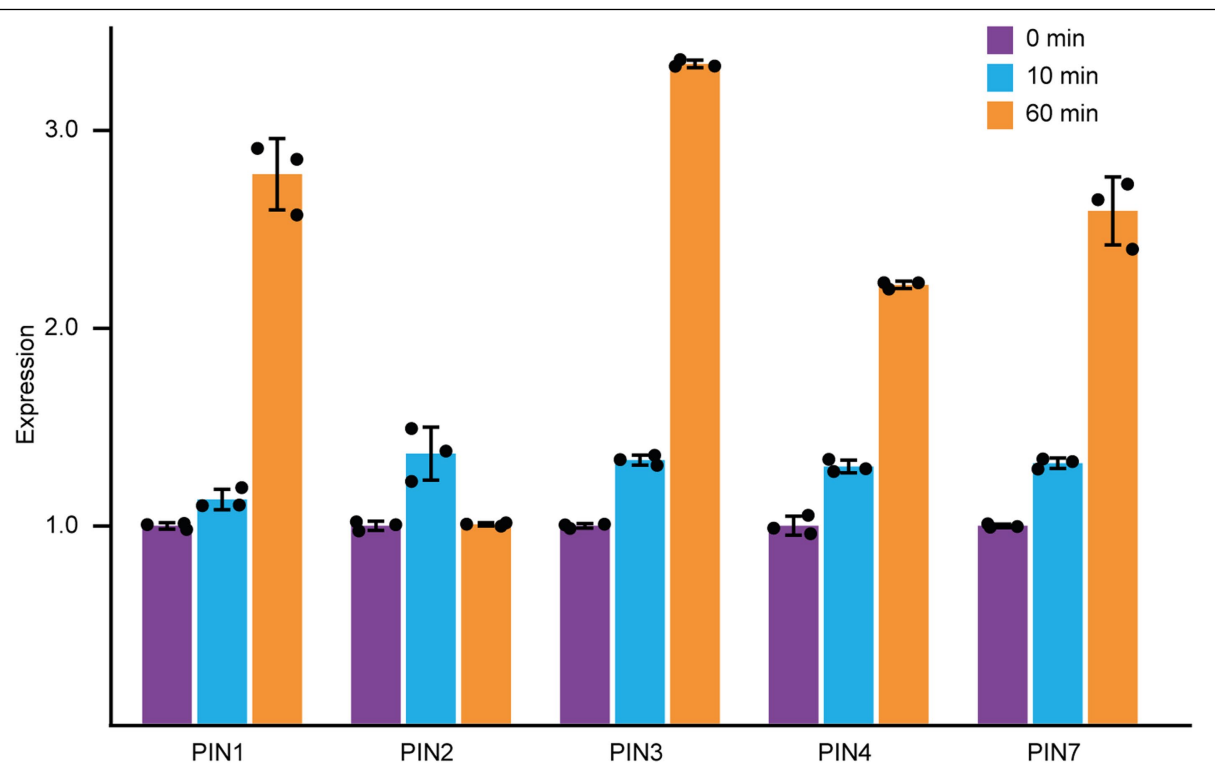

Extended Data Fig. 8 | Quantitative analysis of PINmRNA accumulation in seedlings treated with $10 \mu$ M IAA. Seedlings were kept in MS medium

subjected to analysis by quantitative PCR with reverse transcription. Data are mean \pm s.d. derived from 3 technical replicates, and black dots represent individual values. 


\section{Article}

Extended Data Table 1 | In vivo concentration of indole derivatives as reported in plant extracts. See refs. ${ }^{21,45-49}$

\begin{tabular}{|lcc|}
\hline Compound & Endogenous concentration & Reference \\
\hline Indole-3-acetic acid (IAA) & $100 \mathrm{nM}$ & $45,46,47$ \\
Indole-3-butyric acid (IBA) & $<1 \mathrm{nM}$ & 45 \\
Indole-3-acetonitrile (IAN) & $100 \mathrm{nM}$ & 45,21 \\
Indole & $60 \mathrm{nM}$ & 48 \\
Indole-3-carbaldehyde (ICHO) & $100 \mathrm{nM}$ & 49 \\
4-hydroxyindole-3-carbaldehyde (4-HO-ICHO) & $\sim 300 \mathrm{nM}$ & 49 \\
Indole-3-ethanol (IAEt) & $<1 \mathrm{nM}$ & 45 \\
IAA-glucose (Glc) & $\sim 30 \mathrm{nM}$ & 46 \\
\hline
\end{tabular}


Extended Data Table 2 | Dissociation constants and overview of crystallographic data for TrpR and selected variants

a

\begin{tabular}{|c|c|c|c|c|c|c|}
\hline Variant & Ligand & $\mathrm{K}_{\mathrm{D}}(\mu \mathrm{M})$ & $\Delta \mathrm{H}\left(\mathrm{kcal} \mathrm{mol}^{-1}\right)$ & $-\mathrm{T} \Delta \mathrm{S}\left(\mathrm{kcal} \mathrm{mol}^{-1}\right)$ & $\Delta \mathrm{G}\left(\mathrm{kcal} \mathrm{mol}^{-1}\right)$ & $\mathbf{N}$ \\
\hline \multirow[t]{3}{*}{ TrpR } & $T r p$ & $25.0 \pm 0.7$ & $-12.06 \pm 0.11$ & $5.89 \pm 0.12$ & $-6.18 \pm 0.02$ & $1.06 \pm 0.01$ \\
\hline & $I A A$ & $137.4 \pm 0.5$ & $-10.38 \pm 0.49$ & $5.19 \pm 0.50$ & $-5.19 \pm 0.01$ & $1.20 \pm 0.07$ \\
\hline & $I A N$ & $63.7 \pm 1.7$ & $-11.76 \pm 0.22$ & $6.15 \pm 0.07$ & $-5.62 \pm 0.01$ & $0.98 \pm 0.01$ \\
\hline \multirow[t]{3}{*}{ TrpR-S88Y } & Trp & n.d. & n.d. & n.d. & n.d. & n.d. \\
\hline & $I A A$ & $14.6 \pm 0.1$ & $-12.62 \pm 0.06$ & $6.15 \pm 0.06$ & $-6.48 \pm 0.01$ & $1.05 \pm 0.01$ \\
\hline & IAN & $17.9 \pm 0.6$ & $-11.62 \pm 0.01$ & $5.24 \pm 0.03$ & $-6.38 \pm 0.03$ & $1.04 \pm 0.00$ \\
\hline \multirow[t]{3}{*}{ TrpR-T44L-S88Y } & Trp & n.d. & n.d. & n.d. & n.d. & n.d. \\
\hline & $I A A$ & $7.8 \pm 0.3$ & $-14.73 \pm 0.01$ & $7.88 \pm 0.03$ & $-6.85 \pm 0.03$ & $1.05 \pm 0.01$ \\
\hline & $I A N$ & $19.5 \pm 0.6$ & $-13.29 \pm 0.07$ & $6.97 \pm 0.09$ & $-6.32 \pm 0.01$ & $1.25 \pm 0.00$ \\
\hline \multirow[t]{3}{*}{ TrpR-T44L-T81M-S88Y } & Trp & n.a. & n.a. & n.a. & n.a. & n.a. \\
\hline & $I A A$ & $3.0 \pm 0.1$ & $-18.75 \pm 0.21$ & $11.34 \pm 0.23$ & $-7.42 \pm 0.02$ & $1.05 \pm 0.01$ \\
\hline & $I A N$ & $33.6 \pm 0.2$ & $-13.55 \pm 0.04$ & $7.53 \pm 0.03$ & $-6.02 \pm 0.00$ & $1.17 \pm 0.01$ \\
\hline \multirow[t]{3}{*}{ TrpR-T44L-T81M-N87G-S88Y } & Trp & n.a. & n.a. & n.a. & n.a. & n.a. \\
\hline & $I A A$ & $1.6 \pm 0.0$ & $-20.60 \pm 0.26$ & $12.80 \pm 0.26$ & $-7.81 \pm 0.02$ & $1.10 \pm 0.01$ \\
\hline & $I A N$ & $46.4 \pm 1.7$ & $-16.31 \pm 0.10$ & $10.49 \pm 0.12$ & $-5.82 \pm 0.03$ & $1.19 \pm 0.01$ \\
\hline \multirow{4}{*}{$\begin{array}{l}\text { TrpR-M42F-T44L-T81M-N87G- } \\
\text { S88Y }\end{array}$} & Trp & n.a. & n.a. & n.a. & n.a. & n.a. \\
\hline & $I A A$ & $1.5 \pm 0.1$ & $-19.67 \pm 0.10$ & $11.87 \pm 0.12$ & $-7.82 \pm 0.01$ & $1.11 \pm 0.01$ \\
\hline & $I A N$ & $14.3 \pm 0.4$ & $-13.16 \pm 0.23$ & $6.65 \pm 0.21$ & $-6.51 \pm 0.02$ & $1.21 \pm 0.01$ \\
\hline & $P A A$ & n.a. & n.a. & n.a. & n.a. & n.a. \\
\hline
\end{tabular}

b

\begin{tabular}{|c|c|c|c|c|c|c|}
\hline Variant & Ligand & PDB ID & Resolution & $\mathbf{R}_{\text {free }} / \mathbf{R}_{\mathrm{obs}}{ }^{+}$ & Spacegroup & Reference or source \\
\hline TrpR, tetragonal & TRP & $1 \mathrm{ZT9}$ & 2.0 & 0.219 & P 43 & Lawson, C.L., released 9/2006 (PDB) \\
\hline TrpR, orthorhombic & $T R P$ & $20 Z 9$ & 1.7 & $0.180^{+}$ & P 21212 & Ref. 32 \\
\hline TrpR & $I A A$ & 6EJW & 2.0 & 0.243 & P 43 & This work \\
\hline TrpR-S88Y & IAA & $6 \mathrm{EJZ}$ & 1.9 & 0.218 & P 43 & This work \\
\hline TrpR-S88Y-T44L & IAA & 6ENI & 1.1 & 0.176 & P 212121 & This work \\
\hline TrpR-T44L-T81M-S88Y & IAA & 6EKP & 1.6 & 0.190 & P 212121 & This work \\
\hline TrpR-T44L-T81M-N87G-S88Y & IAA & 6ENN & 1.2 & 0.180 & P 212121 & This work \\
\hline TrpR-M42F-T44L-T81M-N87G-S88Y & IAA & 6ELB & 1.4 & 0.193 & P 212121 & This work \\
\hline TrpR-M42F-T44L-T81I-S88Y & IAA & $6 \mathrm{ELF}$ & 1.8 & 0.217 & P 212121 & This work \\
\hline TrpR-M42F-T44L-T81I-S88Y & IAN & 6ELG & 1.4 & 0.177 & P 212121 & This work \\
\hline
\end{tabular}

a, Dissociation constants of selected variants for TRP, IAA, IAN and PAA as determined by ITC. Errors are calculated from fits of two technical replicates. n.d. indicates that the data was measured but the fit did not converge owing to low affinity, whereas for later variants TRP measurements were omitted altogether (n.a.). The dissociation constants were determined via ITC, at $20^{\circ} \mathrm{C}$ and pH 8.O. Thermodynamic values for enthalpy $(\Delta H)$, entropy $(-T \Delta S)$, and free energy $(\Delta G)$ are shown in kcal mol ${ }^{-1}$. Stoichiometry is given as $N$. Data are mean \pm s.d., derived from 2 technical replicates.

b, Overview of crystallographic data for TrpR and its variants in complex with ligands as indicated. 


\section{Reporting Summary}

Nature Research wishes to improve the reproducibility of the work that we publish. This form provides structure for consistency and transparency in reporting. For further information on Nature Research policies, see our Editorial Policies and the Editorial Policy Checklist.

\section{Statistics}

For all statistical analyses, confirm that the following items are present in the figure legend, table legend, main text, or Methods section.

$\mathrm{n} / \mathrm{a} \mid$ Confirmed

$\square$ The exact sample size $(n)$ for each experimental group/condition, given as a discrete number and unit of measurement

$\square$ A statement on whether measurements were taken from distinct samples or whether the same sample was measured repeatedly

$\triangle$ The statistical test(s) used AND whether they are one- or two-sided

$\square$ Only common tests should be described solely by name; describe more complex techniques in the Methods section.

$\bigotimes \square$ A description of all covariates tested

Х $\square$ A description of any assumptions or corrections, such as tests of normality and adjustment for multiple comparisons

$\triangle$ A full description of the statistical parameters including central tendency (e.g. means) or other basic estimates (e.g. regression coefficient)

AND variation (e.g. standard deviation) or associated estimates of uncertainty (e.g. confidence intervals)

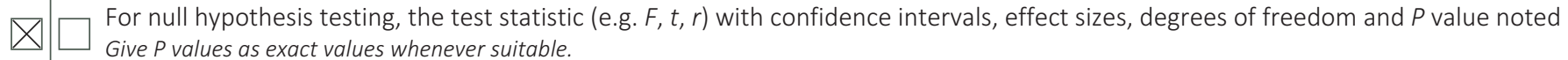

$\bigotimes \square$ For Bayesian analysis, information on the choice of priors and Markov chain Monte Carlo settings

Х $\square$ For hierarchical and complex designs, identification of the appropriate level for tests and full reporting of outcomes

\ $\square$ Estimates of effect sizes (e.g. Cohen's $d$, Pearson's $r$ ), indicating how they were calculated

Our web collection on statistics for biologists contains articles on many of the points above.

\section{Software and code}

Policy information about availability of computer code

Data collection ZEN (Zeiss) / ZEN 2.3 black SP1

MicroCal VPViewer2000 1.04.0011

TA Instruments RunITC 3.4

Data analysis ImageJ 1.53c/ The ImageJ macros used to analyze data are available at "zenodo.org" as "10.5281/

zenodo.4524537" ("Herud_et_al_2021_ImageJ_analysis_macros").

Spectral Unmixing Plugin Version 1.3 (Joachim Walter)

Adaptive Threshold Plugin (by Qingzong Tseng)

ROI Color Coder Plugin (BAR library 1.1.13, by Tiago Ferreira)

MicroCal LLC based on Origin 7.0

TA Instruments NanoAnalyze 3.7.0

XDS BUILT 20170215

XDSCONV BUILT 20170215

Phenix.Phaser 2.6.0

Phenix.refine 1.12_2829

Coot 0.8 .9

For manuscripts utilizing custom algorithms or software that are central to the research but not yet described in published literature, software must be made available to editors and reviewers. We strongly encourage code deposition in a community repository (e.g. GitHub). See the Nature Research guidelines for submitting code \& software for further information. 
Policy information about availability of data

All manuscripts must include a data availability statement. This statement should provide the following information, where applicable:

- Accession codes, unique identifiers, or web links for publicly available datasets

- A list of figures that have associated raw data

- A description of any restrictions on data availability

All processed data generated or analysed during this study are included in this published article and its supplementary information files. Imaging ("Herud_et_al_2021_figures_3 and_4_raw_data") and in vitro source data („Herud_et_al_2021_suppl_figures_S1_S3-S5_S7_and_table_S2_raw_data) are available at "zenodo.org" as "10.5281/zenodo.4524537". The data for the FACS measurements in Fig. 3a and b and Extended data Figures S6 and S7 can be found at "flowrepository.org" as "FR-FCM-Z3FL". Coordinates and structure factors for all reported X-ray crystallography have been deposited as 6EJW, 6EJZ, 6ENI, 6EKP, 6ENN, 6ELB, 6ELF, 6ELG at the "protein data bank" (pdb).

\section{Field-specific reporting}

Please select the one below that is the best fit for your research. If you are not sure, read the appropriate sections before making your selection. $\bigotimes$ Life sciences $\quad \square$ Behavioural \& social sciences $\quad \square$ Ecological, evolutionary \& environmental sciences

For a reference copy of the document with all sections, see nature.com/documents/nr-reporting-summary-flat.pdf

\section{Life sciences study design}

All studies must disclose on these points even when the disclosure is negative.

Sample size Sample sizes were chosen to yield clear results but were limited by the manual processing during the time course studies. Both treated and control samples comprised several seedlings (numbers indicated in the text) in each of which hundreds of nuclei were analysed in the root tip.

Data exclusions No data were excluded from the analysis.

Replication Experiments were repeated at least three times, involving different seedling populations on different days. All attempts at replication were successful.

Randomization Individual 5 days old seedling populations of common ancestry were partitioned into two or three groups that were then treated in different ways.

Blinding No blinding. Microscopic data acquisition was manual, interpretation was automatic involving the ImageJ macro.

\section{Reporting for specific materials, systems and methods}

We require information from authors about some types of materials, experimental systems and methods used in many studies. Here, indicate whether each material, system or method listed is relevant to your study. If you are not sure if a list item applies to your research, read the appropriate section before selecting a response.

Materials \& experimental systems

\begin{tabular}{|c|c|}
\hline $\mathrm{n} / \mathrm{a}$ & Involved in the study \\
\hline$\bigotimes$ & Antibodies \\
\hline$\bigotimes$ & Eukaryotic cell lines \\
\hline Х & Palaeontology and archaeology \\
\hline$\bigotimes$ & Animals and other organisms \\
\hline$\bigotimes$ & Human research participants \\
\hline$\bigotimes$ & $\square$ Clinical data \\
\hline 邓 & $\square$ Dual use research of concern \\
\hline
\end{tabular}

\begin{tabular}{l|l}
\multicolumn{2}{l}{ Methods } \\
\hline n/a & Involved in the study \\
$\square$ & $\square$ ChIP-seq \\
\hline & $\square$ Flow cytometry \\
$\square$ & $\square$ MRI-based neuroimaging
\end{tabular}




\section{Plots}

Confirm that:

$\bigotimes$ The axis labels state the marker and fluorochrome used (e.g. CD4-FITC).

$\bigotimes$ The axis scales are clearly visible. Include numbers along axes only for bottom left plot of group (a 'group' is an analysis of identical markers). $\bigotimes$ All plots are contour plots with outliers or pseudocolor plots.

$\bigotimes$ A numerical value for number of cells or percentage (with statistics) is provided.

\section{Methodology}

Sample preparation

Instrument

Software

Cell population abundance

Gating strategy
Arabidopsis cell-suspension-culture cells were protoplasted and transfected with FP reporter plasmids. The next day the cells were filtered $(100 \mu \mathrm{m})$ and treated with various IAA concentrations following the dilution scheme provided in Extended data Table S4.xlsx, 1 hour before in vivo flow cytometric analysis.

\section{XDP MoFlo Beckman Coulter}

Summit 5.5 (Beckman Coulter) was used for acquisition and analysis; FCS Express v6.06.0033 (deNovo) was used for plotting examples. The FRET-ratio calculation is carefully detailed in the Methods.

Only analysis was performed in the shown region (see Fig3, Fig.S6.).

FSC/SSC gates of starting population marked in Ext data Fig S7a, boundaries between "positive" and "negative" cell populations indicated in Ext data Fig S7b-d

$\bigotimes$ Tick this box to confirm that a figure exemplifying the gating strategy is provided in the Supplementary Information. 